\title{
MEITOIC INSURANCE: DESIGNING A SYSTEM TO STUDY CROSSOVER CONTROL IN YEAST
}

\author{
A Thesis \\ presented to the faculty of \\ California Polytechnic State University, \\ San Luis Obispo \\ In Partial Fulfillment \\ of the Requirements for the Degree \\ Master of Science in Biological Sciences \\ by \\ Kate V. Karfilis \\ June 2010
}


(C) 2010

Kate V. Karfilis

ALL RIGHTS RESERVED 


\section{COMMITTEE MEMBERSHIP}

TITLE: MEIOTIC INSURANCE; DESIGNING A SYSTEM TO STUDY CROSSOVER CONTROL IN YEAST

AUTHOR: Kate V. Karfilis

DATE SUBMITTED: June 2010

COMMITTEE CHAIR: Kenneth Hillers, Ph.D.

COMMITTEE MEMBER: Michael Black, Ph.D.

COMMITTEE MEMBER: Elena Keeling, Ph.D. 


\section{ABSTRACT \\ MEIOTIC INSURANCE; DESIGNING A SYSTEM TO STUDY CROSSOVER CONTROL IN YEAST}

\section{Kate V. Karfilis}

Meiosis is a specialized form of cell division in which haploid gametes are produced from diploid progenitors. This reduction in ploidy results from proper meiotic chromosome segregation and is ensured by crossover recombination events. Given their importance, it is no surprise that crossover formation is regulated in most eukaryotes. Crossover assurance is a regulatory mechanism that helps to ensure that each pair of chromosomes gets at least one crossover during meiosis.

We seek to better understand how crossover assurance works. To do so, we have developed a system in which crossover formation between a pair of chromosomes is restricted to a defined region. If crossover assurance functions in this context, then crossovers should frequently form in this defined region.

Our experiments involve three yeast strains:

Homolog: diploid Saccharomyces cerevisiae. Homologs Homeolog: Diploid S. cerevisiae, but with one copy of III derived from S. paradoxus and one from S. cerevisiae.

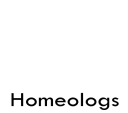
Homo-meolog: The homeolog strain, but with the HIS4 region of the S. paradoxus III replaced with the corresponding $S$. cerevisiae sequence.
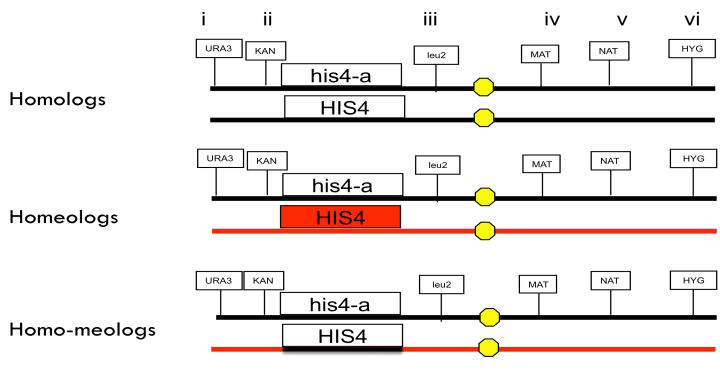
Black = S.cer

S. cerevisiae and S. paradoxus are largely syntenic and have $80-90 \%$ sequence homology. This level of sequence divergence greatly reduces the incidence of meiotic crossing over. Thus, in the Homeolog strain chromosomes III will frequently fail to form crossovers. In the Homo-meolog strain, a defined region of homology surrounding HIS4 (a hotspot for meiotic recombination) exists in a chromosomal context of homeology. In the Homo-meolog strain, crossover assurance should result in a high incidence of crossover formation in the HIS4 region. By comparing the spectrum of meiotic recombination events in the HIS4 region in the three strains, we will gain insight into the means through which crossover assurance is enforced.

These experiments are in the preliminary stage. Strain construction and data collection are ongoing, but our preliminary results demonstrate an elevated incidence of crossing over in the HIS4 region in the homo-meolog strain relative to both the homolog and homeolog strains. Spore viability patterns in the homo-meolog strain are not statistically distinguishable from that of the homolog strain, but are different from that of the homeolog strain. Taken together, these results suggest that the crossovers are targeted to the HIS4 region in the homo-meolog strain, possible through the action of a crossover assurance mechanism. Further analysis of the patterns of recombination in these strains may provide insight into the means through which this regulation is exerted. 


\section{ACKNOWLEDGMENTS}

To Elena Keeling who illuminated the research path in front of me.

To Michael Black who enthusiastically and often threw curves into that path.

To Kenneth Hillers who thankfully dragged me head first and kicking down the path. 
Table of Contents

List of Figures.....................................................................viii

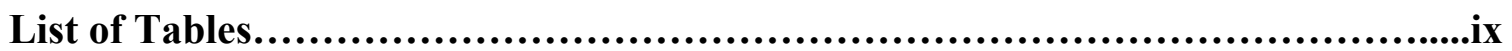
Introduction

Meiosis Overview................................................ $1-2$

Crossover Formation...................................................

Mechanism of Meiotic Recombination.................................. $3-4$

Crossover/Non-crossover Decision.....................................5

Gene conversion and post-meiotic segregation events.....................5-8

Importance of Chromosome Structure for Crossovers.....................8-9

Crossover regulation............................................. $9-15$

Experimental Design...........................................................16-17

\section{Materials and Methods}

Yeast Strains..................................................... 18

Media............................................................18

Genetic and Nutritional Markers...................................... 19

Transformations..................................................... 19

gDNA Isolation.................................................20-21

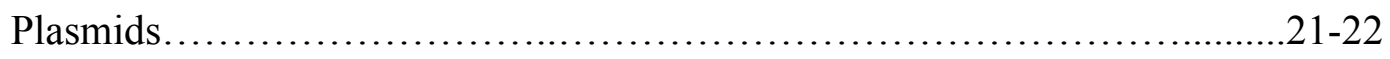

PCR Protocol.....................................................22-23

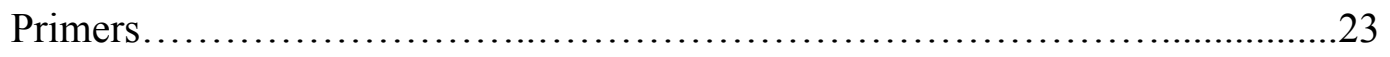

Making Diploid.......................................................25

Tetrad Dissections..................................................25 
Tetrad Analysis.

\section{Data Analysis}

Chi Square Goodness of Fit Test......................................27

Map distance was calculated using the Perkins formula (1949)...............27

\section{Results}

Steps of experimental design....................................28-29

Viability of homeologous chromosomes..............................29-30

Spore Viability of Homeologs and Homo-meologs.......................30-32

Gene conversion events in the Homo-meolog strain......................32-34

Recombination Rates of Homeologs and Homo-meologs...................34-37

Discussion..........................................................................38-47

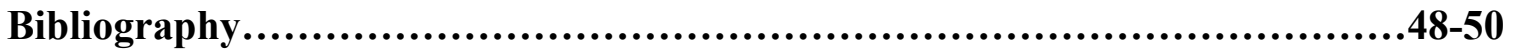




\section{List of Figures}

1- The Holiday Junction Model for Double Strand Break Resolution and

Crossover................................................................................4

2- Gene Conversion Patterns. ........................................................7

3- Reduction of DSBs does not reduce the number of crossovers in parallel

(Martini et al. 2006)............................................................12

4- Planned constructs. ................................................................17

5- Scale depiction of homo-meolog strain.........................................17

6- Strain constructs to date.....................................................28

7- Construction of Homo-meolog strain..........................................30

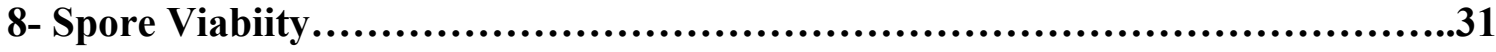

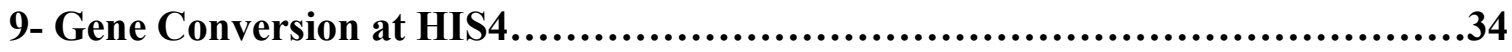

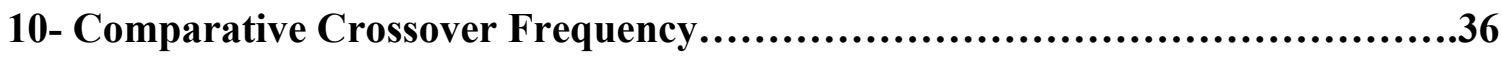

11- Physical and Genetic Maps.....................................................43

12- Pairing and Synapsis Variations between strains. .............................44

13- Double Crossover Formation and NPD Tetrads.............................46 


\section{List of Tables}

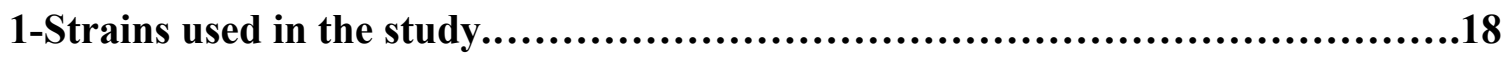

2-Selectable Markers..................................................................19

3-Bacterial Plasmids..........................................................22

4-Primer Descriptions.............................................................24

5- Tetrad Analysis of Diploid Strains..............................................31

6- Mating type test of tetrads........................................................32

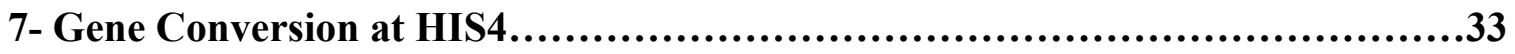

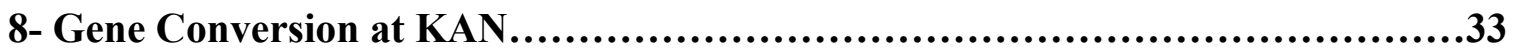

9- Gene Conversion at LEU2 .......................................................33

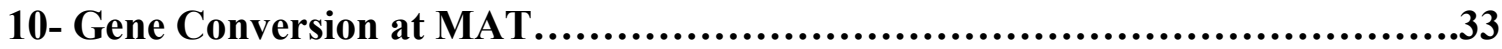

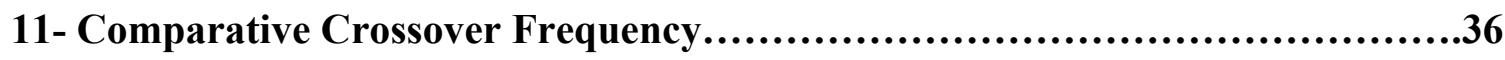

12- Crossover Occurrence Correlation............................................37 


\section{Introduction}

Meiosis Overview

Meiosis is a specialized form of cell division in which haploid cells are produced from diploid progenitors (Roeder, 1997). Meiosis requires two rounds of division to successfully reduce ploidy while maintaining genetic integrity. The two rounds of meiosis are similar in mechanism but distinct in outcome. Meiosis I (MI) is primarily responsible for the separation of homologous chromosomes, resulting in two daughter cells with a reduced ploidy from $2 \mathrm{n}$ to $1 \mathrm{n}$. During Meiosis II (MII), sister chromatids are separated and cell division occurs again to produce four gametes from the initial progenitor. The proper separation of chromosomes (or sister chromatids in MII) and segregation to opposite poles is termed disjunction. In order to ensure faithful disjunction in MI, crossovers between homologous pairs must occur. Crossovers are initiated in the zygotene stage of prophase I, but are not completed until the end of prophase I. Crossovers form physical connections between homologous chromosomes that help to establish proper chromosome attachment to the meiosis I spindle (Nicklas, 1974). In the absence of crossovers, chromosome missegregration can lead to aneuploidy.

Aneuploidy, defined as an abnormal number of chromosomes, frequently results in non-viable offspring. An estimated 10-30\% of all fertilized human eggs are aneuploid, due to unsuccessful reduction and segregation of chromosomes (Hassold and Hunt 2001). Aneuploidy is not well tolerated and is generally embryonic lethal. However, gain or loss of certain chromosomes $(13,18,21$ and the sex chromosomes) can result in developmentally challenged, yet viable, offspring. In yeast, the probability of chromosome mis-segregation for an individual chromosome is approximately 1 in 10,000 (Hassold and 
Hunt 2001). In mammals, however, the probability is much greater. Understanding more about the causes of aneuploidy is of interest to scientists because severe clinical consequences can arise from these mis-segregation events. It has been estimated that almost one-third of clinically recognized spontaneous abortions are aneuploid, and among those fertilizations that survive to term, aneuploidy is the leading genetic cause of developmental disabilities (Hassold and Hunt 2001).

The formation of crossovers is therefore important to avoid aneuploidy and so, the unveiling of the mechanism that regulates these recombination events would be important to better understand the occurrence of crossovers during meiosis. Determining the mechanism that regulates recombination events may also give us a better understanding of why humans are so inefficient at this fundamental reproductive process (Hassold and Hunt 2001).

\section{Crossover Formation}

In order to better understand crossover regulation, the process by which crossovers are formed must first be reviewed. Recombination of genetic material during meiosis is accomplished through the production and completion of a crossover. Crossovers are a process by which homologous chromosomes pair and physically exchange segments of DNA (Fig.1). These junctions require regions of sequence similarity, which are present between homologous chromosomes. Crossover events also establish the necessary tension required for proper chromosomal segregation (Nicklas, 1974). Once the crossover is formed, the meiotic spindle fibers can properly attach to each homolog and pull the chromosomes to separate spindle poles. In many organisms, the importance and necessity 
of proper pairing and crossing over between homologous chromosomes has been demonstrated (Baker et al., 1976).

In order for a crossover to form successfully, a complex series of events must be coordinated. These events include, but are not limited to, the exchange of DNA, exchange of axis and separation and differentiation of sister chromatids (Kleckner, 2006). All of these events must occur with coordinated spatial and temporal timing to ensure proper chromosome alignment and segregation (Kleckner, 2006).

Mechanism of Meiotic Recombination

An understanding of the molecular pathway through which crossovers are formed is fundamental to determining the mechanism of crossover control (Argueso et al., 2004). Recombination, in the form of crossovers, occurs through the repair of double-strand breaks (DSBs), which are generated by the topoisomerase Spo11. The double-strand break repair model, proposed by Szostak et al. (1983) includes unique features such as the repair of double-strand gaps by two rounds of single strand repair and the resolution of two Holliday junctions. Spo11 is a member of a widely conserved family of cleavage proteins throughout all eukaryotic species (Keeney et al., 1997). After homologous chromosomes pair and align, a Spo11, a catalytic topoisomerase responsible for meiotic DNA cleavage activity, nicks one of the homologs (Keeney et al., 1997). The nick creates a small gap (that is subsequently enlarged by endonucleases) in the double-stranded DNA, creating two free 5' ends. These ends undergo resection by exonucleases and leave 3' overhangs for strand invasion. One of the free 3' single strand ends invades the other homologous duplex creating a D-loop. Second end capture, synthesis and ligation can occur and the Holliday 
junctions can be resolved to form crossovers. The alternative to this is that strand displacement occurs (Fig. 1) which will lead to the formation of non-crossovers (Szostak et al., 1983). High-resolution mapping has been used to determine the distribution and frequency of crossovers and non-crossovers in the S. cerevisiae genome and the outcome revealed regions within the genome with preferential distribution of crossovers (Mancera et al., 2008). This unequal distribution may shed insight on crossover regulation by providing key information about the physical location of crossovers throughout a particular genome.

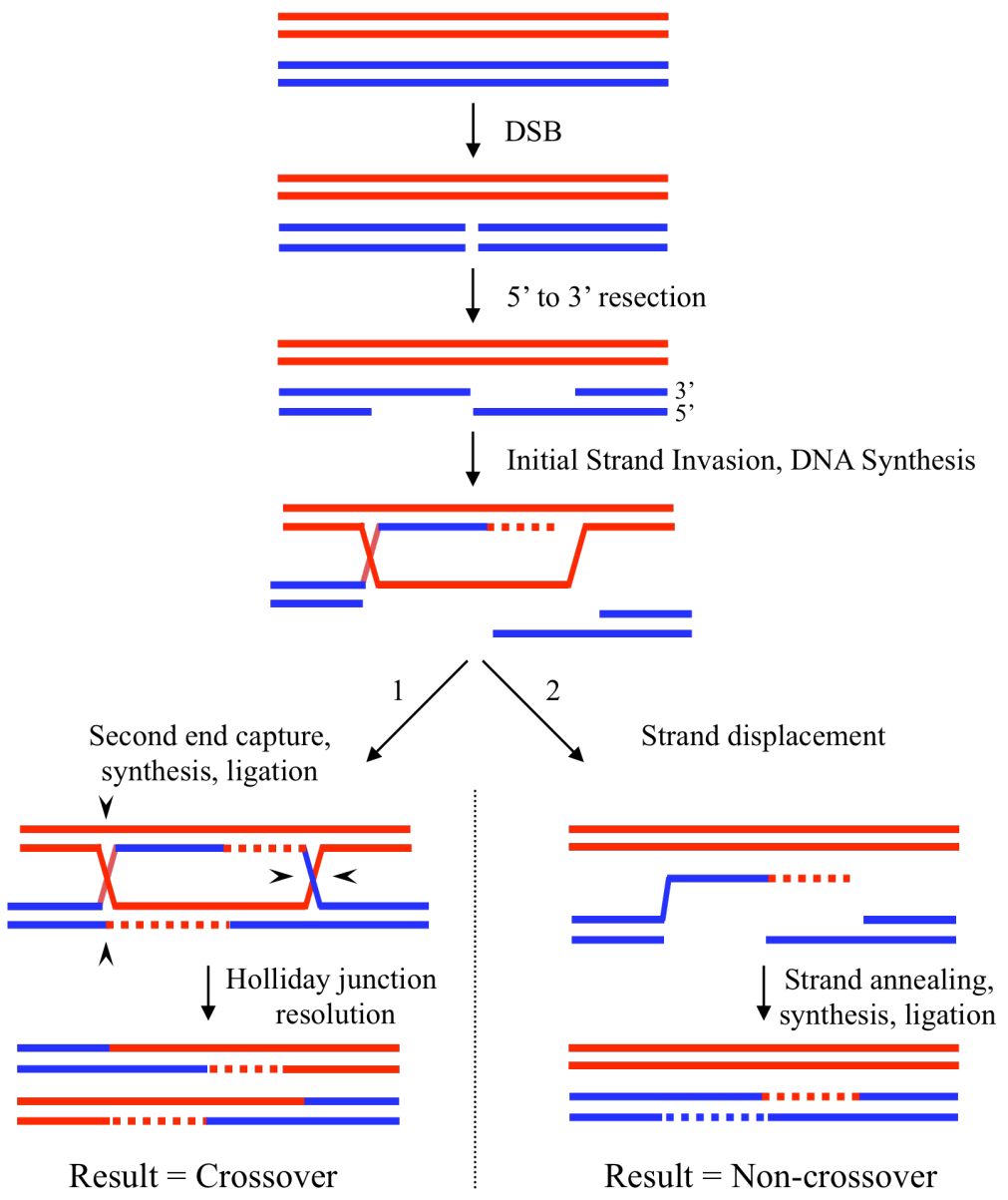

Figure 1: The Holiday Junction Model for Double Strand Break Resolution and Crossover. Homologous chromosomes pair, synapse and then recombination can occur in meiosis I through the formation of a double Holiday junction. The products of the resolved junction are recombinant homologous chromosome. 


\section{Crossover/Non-crossover Decision}

Although DSBs are the precursor to crossovers, not all DSBs that are formed will result in the formation of a crossover. Repair pathways result in the formation of crossovers (CO) or non-crossovers (NCO) (Roig and Keeney 2008). Crossovers are defined as events that involve the reciprocal exchange of large portions of chromosome arms and non-crossovers are defined as the non-reciprocal exchange of smaller segments of chromosomal material (Fig. 1) (Roig and Keeney 2008). The fate of any one DSB can therefore be viewed as a part of crossover control. The ultimate template choice, distribution and type of recombination product produced from DSBs is governed by some still unknown mechanism but some insights are being provided by current genome wide studies (Roig and Keeney 2008). It is however, believed that the pathway choice of $\mathrm{CO} / \mathrm{NCO}$ is made very early in meiotic prophase, at or before Holiday junction resolution (Fig. 1) (Kleckner, 1996).

Gene conversion and post-meiotic segregation events

Gene conversion events occur through the transfer of segments of genetic material from one homologous chromosome to another, and yield an unequal amount of a particular allele. Some, but not all, gene conversion events are associated with recombination in the form of crossing over. Any deviation from the expected ratio of 2:2 allele segregation, as predicted by Mendel, are known as gene conversion events ((Szostak et al., 1983).

Conventional tetrad analysis enables the direct observation of gene conversion events because in yeast, all four meiotic products are packaged together in a tetrad that can be harvested and examined. 
In yeast, aberrant segregation has been observed through gene conversion events (expressed as 3:1 or 1:3 allele segregation pattern) and post-meiotic segregation (PMS) events. PMS is thought to be the result of unrepaired heteroduplex, formed during recombination (Fig. 2) (White et al., 1985). A heteroduplex is defined as double stranded DNA (dsDNA) from different homologous chromosomes, generated from recombination events.

PMS tetrads phenotypically display as one or more sectored colonies derived from a single spore (Detloff et al., 1992). The different cells produced from one parent cell are genetically distinct and therefore can display different growth phenotypes, resulting in a colony which has a section that does not grow like the rest of the cells (Szostak et al., 1983). PMS can be observed as 5:3 or 3:5 segregation patterns, in which the first mitotic division of a haploid nucleus after meiosis yields a pair of cells which vary genetically (Esposito, 1971). If one sectored colony is observed, the segregation pattern is referred to as 5:3 segregation. If two sectored colonies are observed, the segregation pattern is referred to as 4:4 aberrant segregation. Both of these patterns suggest the formation of heteroduplex DNA (Szostak et al., 1983). 


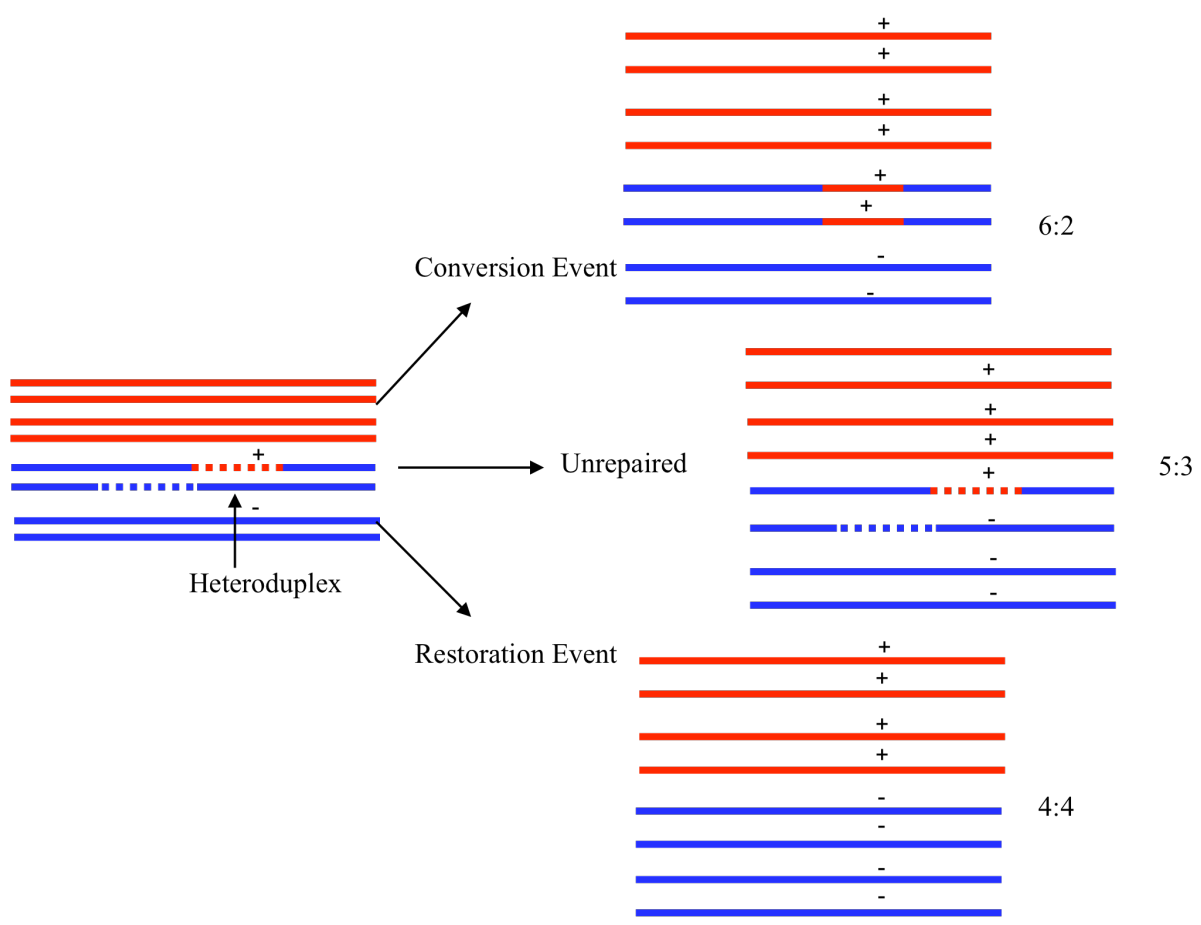

Figure 2: Gene Conversion Patterns. The fate of a heteroduplex formed during recombination can take the above 3 different forms. The heteroduplex can be converted to produce a 6:2 allele segregation pattern, restored to produce a 4:4 pattern or left unrepaired. Note that on the left each line represents double stranded DNA.

Gene conversion models propose that the events are mediated by the heteroduplex formation (Esposito, 1971). Such models view PMS as failure to properly repair mismatches in the heteroduplex after the completion of meiosis. Imagine that the heteroduplex is formed where the mutant donates a strand to the wildtype gene, resulting in a mismatch that can be repaired. This resolution can take two distinct forms: conversion type or restoration type. If removing and replacing the wild-type copy of the gene with the mutant copy can repair the mismatch, it is known as a conversion type event. This will result in a 6:2 gene segregation pattern (Fig. 2). However, if the mutant copy of the gene is removed and replaced with a wild-type copy, a restoration event has occurred and normal Mendelian segregation will follow (4:4 gene segregation pattern, Fig. 2). (Detloff et 
al.,1992). PMS events however, are the result of unrepaired mismatches, which lead to their segregation phenotypes.

In summary, reciprocal exchange is highly correlated with aberrant segregation events and sites of aberrant segregation are associated with areas of high crossover frequency. Therefore a sound understanding of aberrant segregation properties is critical to understanding recombination mechanics (Szostak et al., 1983). Several models have been proposed to explain the molecular mechanism behind recombination and gene conversion events. The most supported models all describe the initiation event as a single or double strand break followed by the generation of heteroduplex DNA (Szostak et al., 1983).

Importance of Chromosome Structure for Crossovers

Hot spots are defined as regions of genomic DNA that experience unusually high levels of recombination (Gerton et al., 1999). Identification and exploration of hot spots in the yeast Saccharomyces cerevisiae have revealed several innate hot spot characteristics. For example, hotspots tend to be located in intergenic, rather than intragenic regions of DNA (Gerton et al., 1999). They also tend to be associated with local double strand breaks, in regions of open chromatin structure that are sensitive to DNase I (Gerton et al., 1999). Hot spots are not easily identified simply on the basis of their DNA sequence alone (Gerton et al., 1999). However, when sequence information is combined with global mapping of DSBs, analysis of recurrent sequences and structural elements can reveal features common to hot spots. Gerton et al. (1999) mapped recombination hot spots and cold spots in the yeast Saccharomyces cerevisiae and identified 177 hot spots. All chromosomes examined in the study contained at least one hot spot and there was a 
significant correlation between the size of the chromosome and the number of hot spots present. Large chromosomes typically have fewer crossovers per kb than smaller chromosomes, and although larger chromosomes have more hot spots, the density and intensity of the hot spots is significantly higher on smaller chromosomes.

The HIS4 locus is a known hot spot in Saccharomyces cerevisiae. This particular hot spot is known as an alpha hotspot, that requires the binding of a transcription factor in the hot spot region. This hot spot has shown a very high frequency of aberrant segregation (up to $50 \%$ non-Mendelian tetrads) but some alleles display few postmeiotic segregation events (Porter et al., 1993). Furthermore, the frequency of gene conversion events occurring at BIK1, which is directly upstream of HIS4, is significantly lower than frequency at the 5' end of HIS4. This suggests that gene conversion events are maximized between the 5'end of HIS4 and the 3' end of BIK1 (Table 2) (Porter et al., 1993).

In summary, it is known that crossovers form via the creation of a DSB and that those breaks do not always result in the creation of a crossover. DSBs and crossovers are not equally distributed and there are genomic regions that receive more DSBs than others, known as hot spots. All of this information is essential in understanding how crossovers are regulated and will help shed light on the mechanism of crossover control.

\section{Crossover regulation}

As previously stated, crossover formation is vital to the viability and meiotic success of an organism. If crossing over occurs at a low frequency, and is not regulated, the probability of chromosomes not receiving a crossover increase. Thus, it is reasonable that the formation of crossovers is regulated. Many studies support the idea that two main 
forms of crossover regulation occur in eukaryotic organisms: crossover interference and crossover assurance (Baker et al., 1976; Jones and Franklin, 2006; Martini et al., 2006)

Crossover interference is the term used to describe the phenomenon that reduces the probability that a second crossover will form in a region neighboring another crossover (Jones and Franklin, 2006). The effects of crossover interference appear to change with distance, such that the regions farther away from the site of the initial crossover experience less interference (Kaback et al., 1999). Non-random spacing of crossovers may be one way to ensure each pair of homologous chromosomes receives at least one crossover. The regulation of the placement of crossovers will ensure that multiple crossovers do not form close to one another and therefore help facilitate that even the smaller chromosomes receive at least one crossover per meiosis.

Furthermore, crossover interference is influenced by chromosome size. Enhanced crossover interference has been reported on larger chromosomes (Kaback et al., 1999). This increased regulatory activity has been correlated with a decrease in the fraction of homologs that fail to crossover. Since all chromosomes are not equal in size, the recombination rates must differ; otherwise, assuming the genome-wide crossover rate, small chromosomes would fail to receive a crossover. In yeast, assuming a genome-wide recombination rate, approximately $5 \%$ of small chromosomes would fail to receive a crossover (Kaback et al., 1999).

Since crossovers are essential to the success of meiosis and yet the formation of crossovers is infrequent (Bascom-Slack et al., 1997), it has been also been suggested that there must be a regulatory mechanism that controls the formation of crossovers in favor of non-crossovers. Crossover assurance is the mechanism that ensures that each pair of 
homologous chromosomes receives at least one crossover in order to successfully complete meiosis. The obligate crossover is a term that has been created to define a crossover that occurs on every pair of homologous chromosomes. This crossover ensures that homologous chromosomes are able to effectively segregate and successfully complete meiosis.

In a seminal study investigating the effects of crossover assurance, a Spol1 allelic series was created and crossover activity was quantified by direct measurement of DSBs in genomic DNA and by frequency of recombination events (Martini et al., 2006). More specifically, the Spo11 gene was mutated several times to create mutants that displayed decreasing amounts of activity. The activity of the enzyme Spo11 was negatively altered in the mutants such that fewer than normal DSBs would be created, and the number of crossovers that resulted was observed. Figure 3 shows that even at approximately $30 \%$ DSB formation, crossover formation remains almost unchanged (approximately 95\%). Chromosome segregation patterns were only significantly altered in the most severe Spo11 mutants, and these effects were most readily revealed by spore viability analysis. Interestingly, despite the reduction in DSBs, crossovers levels remained unchanged. These data suggest that the ratio of crossovers to non-crossovers was altered in favor of crossovers, therefore lending credence to the hypothesis that the primary function of crossover assurance is to create an obligate crossover. This evidence would further suggest that the obligate crossover is a genetically controlled event. Since there appeared to be a threshold level of DSBs under which the depletion of DSBs produced a negative effect on the frequency of crossovers (Fig. 3), it appears crossover assurance cannot act with perfect efficiency. Crossover assurance cannot produce $100 \%$ crossover frequency because of a 
variety of limitations on the system. In order for a crossover to form and be repaired successfully, the proper recombination partner must be present and engaged, and repair of DSBs must be influenced towards the homolog rather than the sister chromatid (Martini et al., 2006).

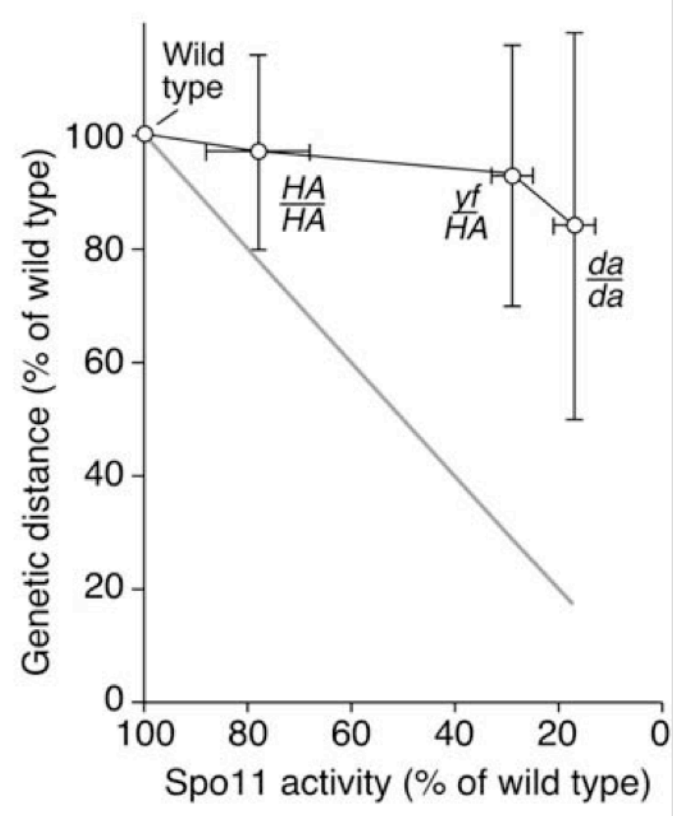

Figure 3: Reduction of DSBs does not reduce the number of crossovers in parallel (Martini et al., 2006). The relationship between DSBs and crossovers does not appear to be completely correlated. However there appears to be a level at which the decrease in DSBs does impede the production of crossovers. If the relationship between DSBs and crossovers was completely linear, a decrease in DSBs would result in an equal decrease in crossovers, represented by the linear line.

The existence of a regulated system of crossover assurance is further supported by the observed segregation fidelity of the sex chromosomes of higher organisms. Human sex chromosomes contain regions of chromosomes that undergo restricted crossing over known as the pseudoautosomal regions (PAR). The PAR is a limited region of homology between otherwise heterologous chromosomes. Pseudoautosomal regions allow the $\mathrm{X}$ and Y chromosome to pair, undergo crossing over and segregate properly during meiosis, while avoiding issues of genetic and structural differences (Mangs and Morris, 2007). 
Although these regions represent a small fraction of the entire length of these chromosomes, they are large enough to allow for crossing over to occur. Therefore, these regions may in fact be well documented, if poorly understood, examples of the effects of crossover assurance (Martini et al., 2006).

In summary, the crossover number per chromosome pair is low in most eukaryotes and the frequency of chromosomes lacking crossovers is lower than would be expected in the absence of some assurance mechanism. Also, crossovers exhibit homeostasis and decreases in DSB frequency do not always lead to a reduction in crossovers, revealing the action of a system that acts to promote the crossover outcome of DSB repair (at the expense of non-crossovers). Finally, crossovers may be subject to chromosome sizedependent regulation and some studies indicate that smaller chromosomes have higher crossover frequencies when compared to larger chromosomes. Taken together, these results could reflect the action of a crossover assurance mechanism. Given this information, our objective was to investigate crossover assurance without altering chromosome size or recombination machinery. Our approach centers on generating a pair of homeologous chromosomes that have a limited region of homology in which crossovers can occur. Using this system, we seek to confirm the presence of a mechanism governing crossover assurance in the budding yeast Saccharomyces cerevisiae.

Yeasts are often used in a variety of experiments to study a wide array of genetic processes because of their ability to be genetically manipulated and the relatively simple analysis of progeny. Furthermore, yeasts are able to reproduce in a haploid or diploid state and most strains, while sharing genetic similarities, also express an array of natural 
phenotypic differences. These differences also contribute to their usefulness in a laboratory setting.

Our hypothesis is that a region of perfect homology between otherwise nonhomologous chromosomes, especially in hot spots, is necessary and sufficient to allow for crossover assurance to act. To address the hypothesis, a system was created to observe and test the effects of crossover assurance on recombination by manipulating the amount of homology available for crossover events to occur, as previously stated. To accomplish this we exploited known crossover incompatibility between particular non-homologous chromosomes, known as homeologs. When DNA sequences of two species differ by more than $10 \%$ they cannot undergo recombination through crossing over and are therefore considered reproductively incompatible. This recombination roadblock makes homeologs ideal for testing the limits of crossover assurance. Homeologous chromosomes exhibit a reduction in genetic exchange and reduced spore viability (Chambers et al., 1996). This is likely caused by a suppression of crossovers.

We will use Saccharomyces cerevisiae and Saccharomyces paradoxus as our study organisms for this project. S. cerevisiae has long been used in the modern world to make breads, wines and other marketable products, and over the past several decades they have also become one of the most widely used model organisms in research labs (Ruderfer, 2006). The ability to save and compare different generations of yeast with their own ancestors, as well as their short generation time and the simplicity of their life cycle and propagation make these species of yeast ideal for studying genetics (Zeyl, 2000). $S$. cerevisiae and S. paradoxus have DNA sequences that differ by 8-20\% (Naumov et al., 1992). These species mate efficiently but less than $1 \%$ of the meiotic products, known as 
spores in yeast, are viable. Those that are viable show a low frequency of genetic exchange and a high degree of chromosome mis-segregation (Chambers et al., 1996). Furthermore, the genomes of S. cerevisiae and S. paradoxus have been sequenced, and crossover hotspots have been identified and mapped in S. cerevisiae (Gerton et al., 1999). 


\section{Experimental Design}

We seek to better understand how crossover assurance works. To do so, we have developed a system where there is a limited region of homology between a pair of chromosomes in which a crossover can occur. If a regulatory mechanism such as crossover assurance exists, then crossovers should frequently form in this defined region. Both copies of chromosome III in the homolog strain are of S. cerevisiae origin. One of these copies will be marked with genetic and nutritional markers along the entire length of the chromosome in order to effectively detect crossover events (Fig. 4). In the homeolog construct however, one copy of chromosome III is of S. cerevisiae origin, while the other is from S. paradoxus (Fig. 4). It has been observed that the frequency of crossing over is significantly reduced in this hybrid circumstance due to sequence divergence between $S$. cerevisiae and S. paradoxus (Chambers et al., 1996). Finally, through the use of genetic engineering, we will replace a portion of the $S$. paradoxus chromosome III with the corresponding sequence from $S$. cerevisiae chromosome III. Thus, we will create a pair of chromosomes with a very limited and defined region in which crossovers can occur (Fig. 5). The region examined will be a hot spot, HIS4. We will call this strain the homo-meolog (Fig. 4). This system will allow us to look at the mechanism of crossover assurance and its effects on crossover frequency.

For the design of the experimental strains, we followed a similar set up as in Shubochkina et al. (2001). We replaced the HIS4/BIK1 region of S. paradoxus with a PCR generated amplicon of the HIS4/BIK1 region from $S$. cerevisiae (Fig.4). We started with one insertion size, and will subsequently increase the region of homology to include other neighboring genes up and down stream of HIS4. 


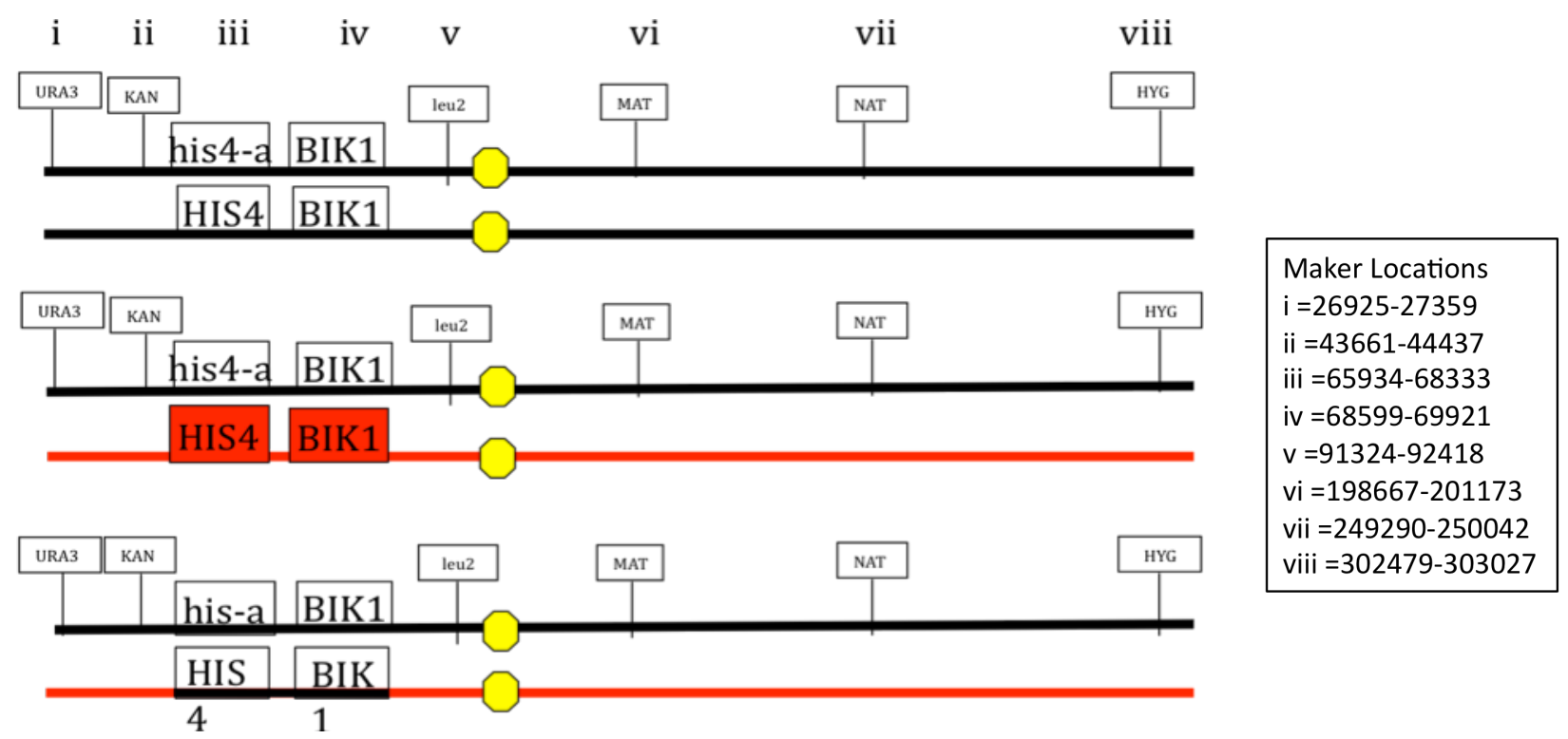

Figure 4: Planned Constructs. S.cer $=$ Black $S$.par $=$ Red. Placement of all markers on S.cerevisiae (From left to right; URA3, KAN, LEU2/leu2 MAT, NAT and HYG.) Each haploid is either mating type a or $\alpha$, and either LEU2+ or leu2- (Table 1) which therefore also serve as markers. A) Homologs of S.cer, positive control B) Homeologs, negative control C) Experimental; Homo-meologs, S.par with region of S.cer homology (Figure not drawn to scale)

Homo-meologs

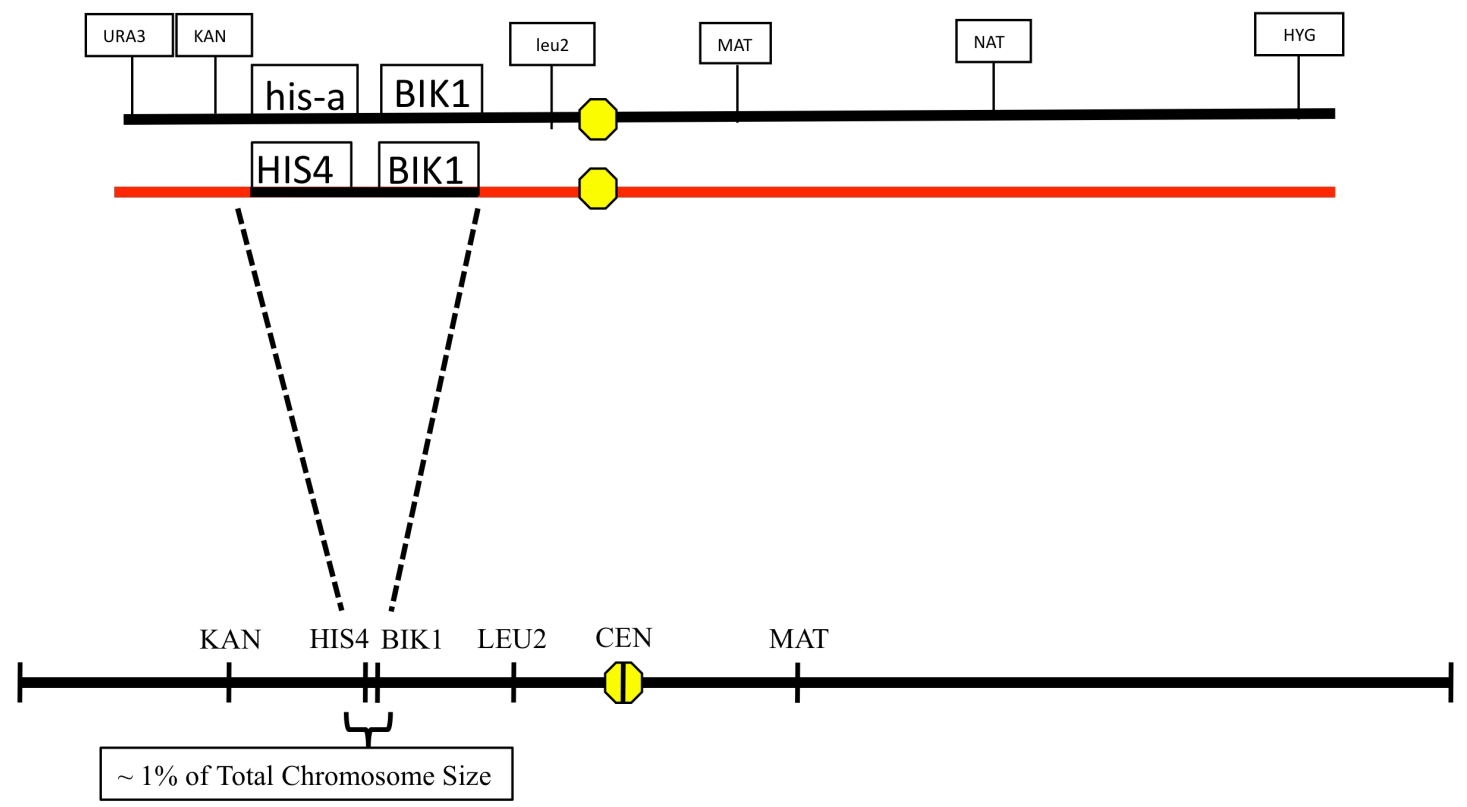

Figure 5: Scale depiction of homo-meolog strain. The homologous insert of the $S$. cerevisiae copy of HIS4/BIK1 is approximately $4 \mathrm{~kb}$ in size. Therefore it represents an extremely small, defined region of the actual total size of chromosome III. 


\section{Materials and Methods}

Yeast Strains

The wildtype yeast strains, Y128, Y597 and S2863, were generously donated by Eva Hoffmann and Rhona Borts. Jonathan Henzel created the KHY130 strain, as described in the methods section under the yeast transformation heading. Kate Karfilis created the constructs KHY246, KHY252 and KHY258 also as described under the yeast transformation heading. See Table 1 for strain genotypes.

Table 1: Strains used in the study

\begin{tabular}{lll}
\hline \multicolumn{1}{c}{ Strain } & \multicolumn{1}{c}{$\begin{array}{c}\text { Chr. III } \\
\text { Species }\end{array}$} & \multicolumn{1}{c}{ Genotype } \\
\hline Y128 & S. cerevisiae & MATa HIS4-HhaI leu2-R1 met13-2 lys2-c ura3-1 \\
Y597 & S. paradoxus & MAT $\alpha$ ade1-1 ura3-Nco cyh2 ${ }^{\mathrm{R}}$ met13-2 kar1-13 \\
S2863 & S. cerevisiae & MAT $\alpha$ trp5-1 CyhR lys2-c ade1-1 ura3-1 his4-ATC \\
KHY 130 & S. cerevisiae & MATa HIS4-HhaI leu2-R1 met13-2 lys2-c ura3-1HYG ${ }^{\mathrm{R}}$ \\
KHY 246 & S. paradoxus & Y597 with HIS4 region replaced with URA3 \\
KHY 252 & S. cerevisiae & MATa his4-ATC leu2-R1 met13-2 lys2-c ura3-1KAN ${ }^{\mathrm{R}}$ \\
KHY 258 & S. paradoxus & KHY246 with URA3 replaced with S.cerevisiae copy of HIS4-BIK1 \\
\hline
\end{tabular}

Media

All yeast strains were maintained in $100 \mathrm{~mm}$ Petri dishes that contained YPAD medium (1\% Yeast Extract, 2\% peptone, 2\% dextrose, $1.75 \%$ agar and $0.004 \%$ adenine sulfate). Synthetic dropout media (SD) was used to select for certain auxotrophic strains, specifically the URA3 intermediate of $S$. paradoxus and the transformant of $S$. cerevisiae containing the URA3 marker on the left end of chromosome III. YPAD media plus the addition of the appropriate antibiotic (Table 2) was also used to select for antibiotic resistant transformants. LB media, with the appropriate selectable marker, was used to grow bacterial plasmids harbored in E. coli. 
Genetic and Nutritional Markers

Antibiotic resistances and nutritional markers were added to chromosome III of $S$. cerevisiae by PCR-mediated gene disruption. Genes were amplified from bacterial plasmids with primers that contained long tails of homology to the $S$. cerevisiae genome for directed insertion. Putative transformants were grown on selective media, either SD single drop out media or YPAD + antibiotic.

Table 2: Selectable Markers

\begin{tabular}{ccccc}
\hline Marker Name & Resistance & Gene Replacement & $\begin{array}{c}\text { Location of Gene } \\
\text { Replaced }\end{array}$ & Dosage of Stock \\
\hline URA3 & Uracil & YCL056C & $26925-27359$ & - \\
KAN & G418 & YCL047C & $43661-44437$ & $100 \mu \mathrm{L}$ of $100 \mathrm{mg} / \mathrm{mL}$ \\
HIS4 & Histadine & YCL030C & $65934-68333$ & - \\
BIK1 & - & YCL029C & $68599-69921$ & - \\
LEU2 & Leucine & YCL018W & $91324-92418$ & - \\
MAT & - & - & $198667-201173$ & $100 \mu \mathrm{L} \mathrm{of} 25 \mathrm{mg} / \mathrm{mL}$ \\
NAT & Nourseothicin & YCR076C & $249290-250042$ & $200 \mu \mathrm{L}$ of $30 \mathrm{mg} / \mathrm{mL}$ \\
\hline
\end{tabular}

Yeast Transformations

Yeast cells were grown overnight $(8-12$ hours $)$ at $30^{\circ} \mathrm{C}$ shaking in $5 \mathrm{~mL}$ of YPAD liquid media. $25 \mathrm{~mL}$ of fresh YPAD media was then inoculated with $750 \mu \mathrm{L}$ to $1 \mathrm{~mL}$ of the overnight culture and grown at $30^{\circ} \mathrm{C}$ shaking until the optical density at $600 \mathrm{~nm}$ of the solution reached 0.4-0.6. Cells were spun down in $50 \mathrm{~mL}$ conical tubes for 3 minutes at 3000 RPM and then the supernatant was decanted. Pellets were resuspend in $25 \mathrm{~mL}$ of $\mathrm{diH}_{2} \mathrm{O}$, and spun again as before. Again, the supernatant was decanted and the pellets were resuspsend in $10 \mathrm{~mL}$ of $\mathrm{Li}-\mathrm{T}$ and spun again. The final pellet was resuspend in $1 \mathrm{~mL}$ of $1 \mathrm{M}$ LiOAc. This solution was transferred to a microcentrifuge tube and spun at max speed for 30 seconds. Supernatant was decanted and the remaining cells were resuspended in $100 \mathrm{mM}$ LiOAc such that each transformant gets $50 \mu \mathrm{L} .50 \mu \mathrm{L}$ aliquots were transferred to 
fresh microcentrifuge tubes and the centrifugation was repeated. A master mix was made for each transformant as follows; $240 \mu \mathrm{L} 50 \%$ PEG 3350, $36 \mu \mathrm{L} 1 \mathrm{M} \mathrm{LiOAc}$ and $50 \mu \mathrm{L}$ boiled salmon sperm. The pellet was resuspend in $325 \mu \mathrm{L}$ of the master mix and then $25-$ $30 \mu \mathrm{L}$ of transforming DNA was added. Cells were incubated for 15 minutes at room temperature and then $8 \mu \mathrm{L}$ of $60 \%$ glycerol was added while mixing gently. Cells were incubated again for 30 minutes at room temperature. After the incubation, cells were heat shocked at $42 \mathrm{C}$ for 10 minutes, then spun down for 1 minute at $6000 \mathrm{rpm}$. Finally cells were resuspended in $100 \mu \mathrm{L} \mathrm{diH}{ }_{2} 0$ and plated for auxotrophic transformants. Antibiotic resistance marker transformations were outgrown in $5 \mathrm{~mL}$ YPAD cultures overnight, then plated on YPAD with the appropriate antibiotic (Table 2).

gDNA Isolation

Genomic DNA was isolated from yeast through one of two methods; Genomic Extraction using Sorbitol and Genomic Extraction using Glass Beads. The protocols yield comparable quantities of gDNA.

Soribtol Extraction

Yeast cultures were grown in $5 \mathrm{~mL}$ YPAD broth overnight at $30^{\circ} \mathrm{C}$ with shaking and spun down in microcentrifuge tubes at 3000 RPM for 2 minutes after sufficient growth was confirmed in the test tube. After decanting the supernatant, the pellet was resuspended in $500 \mu \mathrm{L} 1 \mathrm{M}$ sorbitol. $15 \mu \mathrm{L}$ DTT and $10 \mu \mathrm{L} 100 t$ zymolyase was added to the cells and the solution was incubated with shaking for 1 hour at $37^{\circ} \mathrm{C}$. After incubation, $200 \mu \mathrm{L}$ TE and $70 \mu \mathrm{L} 10 \%$ SDS was added and the cells were incubated at $65^{\circ} \mathrm{C}$ for 10 minutes. Post incubation $350 \mu \mathrm{L} 5 \mathrm{M}$ potassium acetate was added and the tubes were inverted 6 times 
and iced for 30 minutes. The cells were spun down 6 minutes at max speed. Add no more than $650 \mu \mathrm{L}$ of supernatant to prepared $2 \mathrm{~mL}$ microcentrifuge tubes containing $1 \mathrm{~mL}$ isopropanol and $200 \mu \mathrm{L} 5 \mathrm{M}$ ammonium acetate. Spin at $4000 \mathrm{RPM}$ for 1 minute and discard supernatant completely. Pellets were dried in a vacuum centrifuge and resuspend in $300 \mu \mathrm{L}$ TE buffer with $1 \mu \mathrm{L}$ RNase $(10 \mathrm{mg} / \mathrm{mL})$ for storage in the $-20^{\circ} \mathrm{C}$ freezer.

Extraction using Glass Beads

Yeast cultures were grown in $5 \mathrm{~mL}$ YPAD broth overnight at $30^{\circ} \mathrm{C}$ with shaking and spin down in microcentrifuge tubes at max speed for 1 minute after sufficient growth was confirmed in the test tube. After decanting most of the supernatant, 0.3 grams of 425600 micron acid-washed glass beads were added to the cells along with $200 \mu \mathrm{L} \mathrm{X-2-17}$ solution ( $0.2 \% 0.5 \mathrm{M}$ EDTA pH 8.0, $1 \% 1 \mathrm{M}$ Tris $\mathrm{pH} 8.0,5 \% 5 \mathrm{M} \mathrm{NaCl}, 1 \% \mathrm{SDS}, 2 \%$ Triton X-100)) and $200 \mu \mathrm{L}$ phenol:chloroform. Phenol extraction was preformed 3 times, saving the aqueous layer each time. DNA was precipitated with 0.1 volumes of $3 \mathrm{M}$ sodium acetate $\mathrm{pH} 5.2$ and 2 volumes of $95 \%$ ice cold ethanol and incubate at $-20^{\circ} \mathrm{C}$ for 10 minutes. The final solution was spun at max speed for 10 minutes and decanted. Then the pellet was washed with $70 \%$ ice cold ethanol by spinning for 1 minute at max speed and decanting supernatant. The pellet was dried in a vacuum centrifuge and then resuspend in $50 \mu \mathrm{L}$ TE buffer with $0.2 \mu \mathrm{L}$ RNase $(10 \mathrm{mg} / \mathrm{mL})$ for storage at $-20^{\circ} \mathrm{C}$ freezer.

Plasmids

Bacterial plasmids were used to provide the antibiotic resistance genes (Table 3). Minipreps ( $5 \mathrm{~mL}$ starting culture) and maxipreps (50mL starting culture) were used to isolate the desired DNA. Bacteria were grown at $37^{\circ} \mathrm{C}$ shaking overnight ( $8-12$ hours) in LB media plus selective antibiotic. Cells were pelleted in a microcentrifuge tube for 3 
minutes at maximum speed. Supernatant was decanted and the cells were resuspended in $100 \mu \mathrm{L}$ of Solution I (50 mM glucose, $25 \mathrm{mM}$ Tris-Cl $\mathrm{pH}$ 8.0, $10 \mathrm{mM}$ EDTA) and then $200 \mu \mathrm{L}$ of fresh Solution II (0.2 M NaOH, 1\% SDS) was added. The solution was mixed by inverting the microcentrifuge tube 6 times. $150 \mu \mathrm{L}$ of cold Solution III ( $5 \mathrm{M}$ potassium acetate) was then added and mixed again by inversion. Tubes were placed on ice for 10 minutes then spun for 10 minutes at max speed. Supernatant was finally decanted into a fresh tube.

Table 3: Bacterial Plasmids

\begin{tabular}{ccc}
\hline Name & Size (in bp) & Selectable Marker $^{{ }^{2}}$ \\
\hline pAG25 & 3704 & Nouresothricin $^{\mathrm{R}}$ \\
pAG32 & 4160 & Hygromycin $^{\mathrm{R}}$ \\
pAG60 & 3944 & URA3 $^{\mathrm{R}}$ \\
pUG6 & 4009 & Kanamycin $^{\mathrm{R}}$ \\
\hline
\end{tabular}

PCR

Polymerase chain reaction (PCR) mediated gene disruption was used to insert genetic markers onto chromosome III of the Y128 strain and to insert the S.cerevisiae copy of HIS4 into chromosome III of the Y597 strain. Template DNA for PCR was obtained either by gDNA isolation and colony PCR from yeast or bacterial plasmid prep. Both methods of yeast gDNA isolation yielded DNA suitable for PCR, however gDNA extractions were more reliable. PCR conditions for GoTaq reactions all contained 2 minutes at $94^{\circ} \mathrm{C}, 30$ seconds at $94^{\circ} \mathrm{C}$, then 30 cycles of 30 seconds at the specific annealing temperature (Table 4), 30 seconds for each $500 \mathrm{bp}$ at $72^{\circ} \mathrm{C}$, followed by another 4 minutes at $72^{\circ} \mathrm{C}$ and an indefinite hold at $4{ }^{\circ} \mathrm{C}$. All PCR conditions for Phusion reactions contained 30 seconds at $98^{\circ} \mathrm{C}, 10$ seconds at $98^{\circ} \mathrm{C}$, then 30 cycles of 30 seconds at annealing 
temperature (Table 4), 15 seconds per $500 \mathrm{bp}$ at $72^{\circ} \mathrm{C}$, followed by another 5 minutes at $72^{\circ} \mathrm{C}$ and a hold at $4^{\circ} \mathrm{C}$.

PCR reactions contained the following components: $\mathrm{diH}_{2} \mathrm{O}-7.7 \mu \mathrm{L}, 5 \mathrm{x}$ GoTaq buffer $-3 \mu \mathrm{L}$, GoTaq $-0.3 \mu \mathrm{L}$, dNTPs $-0.18 \mu \mathrm{L}, 25 \mathrm{mM} \mathrm{MgCl} 2-0.9 \mu \mathrm{L}, 10 \mathrm{mM}$ Forward primer $-1 \mu \mathrm{L}, 10 \mathrm{mM}$ Reverse primer $-1 \mu \mathrm{L}$.

Primers

Several sets of primers were designed and used in this study and their sequences can be found in Table 4. For each marker added, a primer set was designed to insert the desired resistance marker and then a subsequent primer set was designed to test for the presence of that marker after transformation. Insertion primers were constructed with two distinct segments. The 3 ' end is comprised of a short region (18-20bp) of homology to the plasmid conferring the resistance marker desired. The 5' end incorporates a long region (40-60bp) of homology to the specific desired location of insertion on chromosome III. The use of the long flanking homologous regions aided in the direction and efficiency of insertion of the transforming DNA. 


\begin{tabular}{|c|c|c|c|c|}
\hline & Name & Sequence & $\begin{array}{l}\text { Annealing } \\
\text { Temp. } \\
\left({ }^{\circ} \mathrm{C}\right)\end{array}$ & $\begin{array}{l}\text { Extension } \\
\text { Time } \\
(\min )\end{array}$ \\
\hline 1 & $\begin{array}{l}\text { YCR101C } \\
3, \\
\text { YCR101C } \\
5\end{array}$ & $\begin{array}{l}\text { ctaaatattcttctgatgagggtatagttaaagccctccatgactccccacggtcgtcggtaatgtagaa } \\
\text { GCATAGGCCACTAGTGGATCTG } \\
\text { aaacatcatttaatctagtgagttttgatgattccaacacttctatcagattagatggttggggggttgtGA } \\
\text { TTTAGGTGACACTATAGAACGC }\end{array}$ & 61 & $2: 00$ \\
\hline 2 & $\begin{array}{l}\text { YCR101C } \\
\text { Test F } \\
\text { YCR101C } \\
\text { Test R }\end{array}$ & $\begin{array}{l}\text { CCAAAAGTAACGGAGGCTCC } \\
\text { GCTTTAAGTTGTTATATCGTCGTC }\end{array}$ & 56 & $2: 15$ \\
\hline 3 & $\begin{array}{l}\text { YCL056C } \\
3, \\
\text { YCL056C } \\
5,\end{array}$ & $\begin{array}{l}\text { ggagctgggcttacagaactaaattcgttcaagcataaaaGCATAGGCCACTAGTGG } \\
\text { ATCTG } \\
\text { ggagatgaataaaagtaaaggaagaagaaaagaaagttgaGATTTAGGTGACACTAT } \\
\text { AGAACGC }\end{array}$ & 65 & $1: 30$ \\
\hline 4 & $\begin{array}{l}\text { YCL056C } \\
\text { Test F } \\
\text { YCL056C } \\
\text { Test R }\end{array}$ & $\begin{array}{l}\text { cgccectttgctcttattgc } \\
\text { tcagactcttactctgcegg }\end{array}$ & 55 & $2: 15$ \\
\hline 5 & $\begin{array}{l}\text { SpHis+Bik- } \\
\text { URA3 3' } \\
\text { SpHis- } \\
\text { URA3 5 }\end{array}$ & $\begin{array}{c}\text { tattttgatatatatatattttttcgttagaatgtccgaatttccaagtttgaaataacGCATAGGCC } \\
\text { ACTAGTGGATCTG } \\
\text { atatcatactacaagtgcgctgtgtaatagtaacataatagttaacaattttttgaataGATTTAGG } \\
\text { TGACACTATAGAACGC }\end{array}$ & 62 & $1: 15$ \\
\hline 6 & Spar F & ctcctcctgctaccgctttc & 55 & 4:00 \\
\hline 7 & $\begin{array}{l}\text { BIK } 1 \mathrm{R} \\
\text { YCL0 } 047 \mathrm{C} \\
3 \\
\text { YCL047C } \\
5\end{array}$ & $\begin{array}{c}\text { CCTTTCTTCTGTAGGTTGGG } \\
\text { gttgctatgttattgttttaagattactagtctagtataacagaaagttttttgttccGCATAGGCC } \\
\text { ACTAGTGGATCTG } \\
\text { ttggaaaatggacacttaatcaatgacggttgatagcagtacgtgcactagcagactaatGATTTA } \\
\text { GGTGACACTATAGAACGC }\end{array}$ & 61 & $1: 30$ \\
\hline 8 & $\begin{array}{l}\text { YCL047C } \\
\text { Test F } \\
\text { YCL047C } \\
\text { Test R }\end{array}$ & $\begin{array}{l}\text { gaagttgaaatcccaatggc } \\
\text { ggacaaacccatcaaatgg }\end{array}$ & 55 & $2: 00$ \\
\hline 9 & $\begin{array}{l}\text { YCR076C } \\
3 \text {, } \\
\text { YCR076C5 }\end{array}$ & $\begin{array}{l}\text { tcaataaattctttccttccctttcgacatagctatgtctgtatactatctaaaaactaaatatcacaataaac } \\
\text { aaagGCATAGGCCACTAGTGGATCTG } \\
\text { atataagtttgttatccttttttttcataaaaaacccgtattagcatcaataataccaccatagtaacgcaag} \\
\text { agGATTTAGGTGACACTATAGAACGC }\end{array}$ & 61 & $1: 30$ \\
\hline 10 & $\begin{array}{l}\text { YCR076C } \\
\text { Test F } \\
\text { YCR076C } \\
\text { Test R }\end{array}$ & $\begin{array}{l}\text { ggggttggtgtatcgcgatg } \\
\text { cgttatagagtctaccggcg }\end{array}$ & 55 & $2: 00$ \\
\hline 11 & $\begin{array}{l}\text { His4-RRP7 } \\
\text { Bik1-RNQ1 }\end{array}$ & $\begin{array}{c}\text { CTCATATCGTTTGACGTGATATCAATTCTGATAACTATCAGTT } \\
\text { AATCAATAATTGATACccactcttgctactacctc } \\
\text { ATCCAAATATTTTAAAAGGCAGCACAGACTTTACAAAGCCAT } \\
\text { TCTTACATTGATATTTAAcaaaaacgttggacgagac }\end{array}$ & 60 & $2: 00$ \\
\hline 12 & $\begin{array}{l}\text { S.cer spec F } \\
\text { S.par spec } \\
\text { F } \\
\text { Common R }\end{array}$ & $\begin{array}{c}\text { cccactcttgctactacctc } \\
\text { CTCCTCCTGCTACCGCTTTC } \\
\text { TGTGACGGTTACGAAGAAGC }\end{array}$ & 55 & $0: 45$ \\
\hline
\end{tabular}

* Note when both capital and lower case letters are present the primers are two-tailed and therefore contain regions from two different sources, reflected by the change in letter type. 


\section{Making Diploids}

Using a sterile wooden stick, patches of haploid strains were plated onto opposite ends of a YPAD plate and incubated at $30^{\circ} \mathrm{C}$ for 24 hours. Next the plate was replica printed using sterile velvet onto a double drop out plate, to help select for the diploid cells. The plate was again incubated for 24 hours. The cells were then replica printed again to YPAD and incubated for a final 24-hour period.

Tetrad Dissection

To induce meiosis, diploid strains were streaked together in the middle of a YPAD plate and incubated at $30^{\circ} \mathrm{C}$ for 24 hours. Then the fresh cells were transferred to sporulation (SPO) media by replica printing using sterile velvet. The SPO plates were then also incubated at $30^{\circ} \mathrm{C}$ for $2-5$ days depending on the strains. Samples were taken from the SPO plates and examined under a standard light microscope for a high percentage of tetrads. When there were a sufficient proportion of tetrads present in the sample, dissection plates were prepared. Cells from the SPO plate were sampled with a wood stick and added to a solution of $5 \mu \mathrm{L}$ of $\mathrm{diH}_{2} 0$ and $5 \mu \mathrm{L}$ of $100 \mathrm{mg} / \mathrm{mL}$ zymolyase for 10 minutes. A streak on a fresh, and very smooth YPAD plate was made on the left hand side and tetrads were dissected using a Singer micromanipulator dissection microscope. Approximately 18-20 tetrads would be collected per dissection plate. Following dissection, plates were incubated at $30^{\circ} \mathrm{C}$ for $24-48$ hours to allow adequate growth for analysis. 
Tetrad Analysis

After incubation, dissected plates were replica printed to selective media. Four spore viable tetrads were further characterized as parental ditype, non-parental ditype and tetra-type. Parental means all four spores have the exact parental markers, non-parental tetrads would therefore have different marker arrangements than the parental strains and tetratypes would have two spores with parental markings and two spores with non-parental markings. Tetrad characterization was accomplished by observing the combination of genetic markers present in the spores as determined by growth on appropriate selective media (Papazian, 1952). 


\section{Data Analysis}

Chi Square Goodness of Fit Test:

$$
\chi^{2}=\sum(\mathrm{o}-\mathrm{e})^{2} / \mathrm{e}
$$

Where o indicates the actual collected value counts and e represents the expected counts based on a previously determined ratio. $\chi^{2}$ analysis was run using the statistical software, Minitab. Two separate 2x5 Fisher Exact analyses were conducted between the Homologs/Homeologs as well as the Homologs/Homo-meologs.

Map distance was calculated using the Perkins formula (1949):

$$
\mathrm{cM}=(100(6 \mathrm{~N}+\mathrm{T})) /(2(\mathrm{P}+\mathrm{N}+\mathrm{T}))
$$

Where $\mathrm{P}$ indicates parental ditype (all four spores have parental marker arrangement), $\mathrm{T}$ indicates tetratype (two spores have parental marker arrangement, two spores do not) and $\mathrm{N}$ represents non-parental ditypes (all four spores have non-parental marker arrangement). Map distance is represented in centiMorgans (cM), a unit of measurement for assessing genetic linkage of alleles on a single chromosome. One $\mathrm{cM}$ is equivalent to a single map unit and is measured as the chance that a crossover between them will separate two markers on the same chromosome. 


\section{Results}

The experimental system was accomplished in 3 main steps, as described below.

1. Mark S. cerevisiae chromosome III to detect crossover events

To date, the S. cerevisiae, Y128 (Table 1), chromosome III was marked with Kanamycin (KAN) as seen below in Figure 5. To do this we utilized PCR to amplify the insert from pUG6 (Table 3) and then conducted a yeast transformation (see Materials and Methods) with the PCR product as the transforming DNA. The insertion was confirmed by gel electrophoresis. In the future, we will add the remaining markers to the $S$. cerevisiae chromosome III: [hygromycin (HYG), and nourseothicin (NAT) resistances and uracil (URA3)] (Fig. 4). These additional markers will allow for more accurate mapping of crossover events in segments along the length of the entire chromosome.

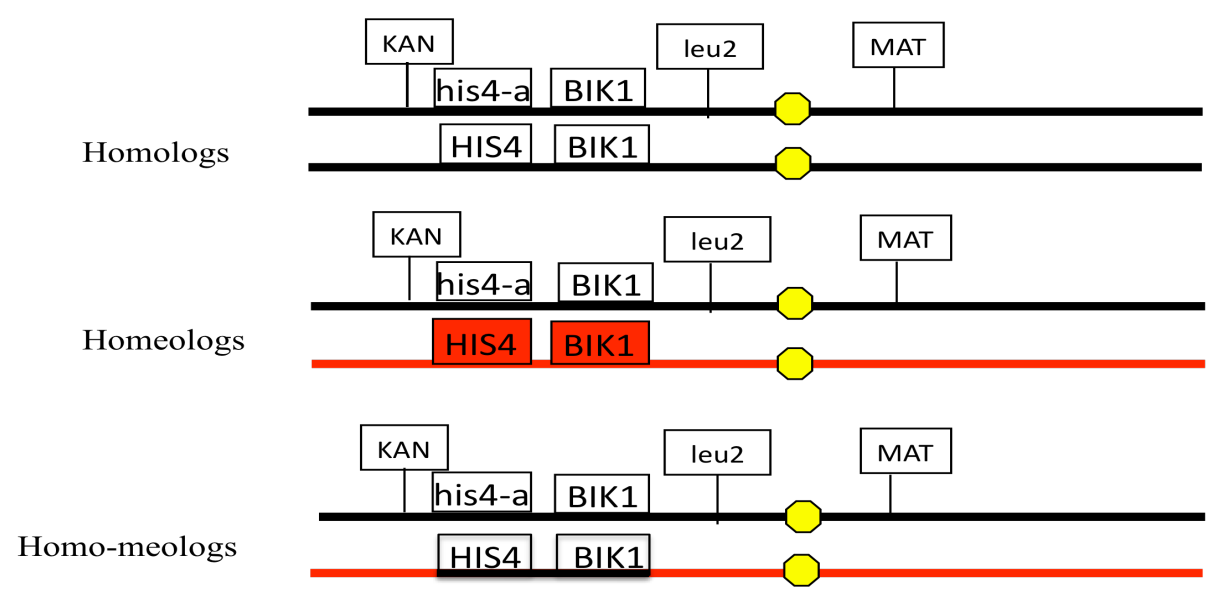

Figure 6: Strain constructs to date. The only marker that has been added to date is Kanamycin. Strain construction is ongoing, and the preliminary results are based on the above constructs. Homologs $=$ Y128 $\alpha$ x KHY252, Homeologs $=$ Y597 x KHY252 and Homo-meologs $=$ KHY252 x KHY258 (Table 1).

2. Create his4- mutant S. cerevisiae 
Through mating and tetrad dissection, we created a his4- mutant version of the marked S. cerevisiae strain. The S2863 strain, which contained the mutant copy of HIS4 was backcrossed to the marked version of Y128 and a haploid was selected based on the markers present to create the strain KHY252 (Table 1).

3. Create homeologous chromosomes with a region of homology by inserting HIS4 region of S. cerevisiae into S. paradoxus

The plasmid vector pAG60, which contains the URA3 marker, was used to initially insert a selectable gene of interest into the S. paradoxus strain (Fig. 7) through PCRmediated gene replacement. Transformants were grown on the selectable media, SD-URA and then colonies that grew were confirmed further with PCR. After creating the $S$. paradoxus strain with the URA3 replacement (KHY246), we then replaced the URA3 gene and the native S. paradoxus BIK1 with an S. cerevisiae copy of our gene of interest, HIS4 and BIK1 (Fig. 7). This was achieved using the same methods as described above. This construction scheme is used to allow for the efficient selection of each transformant. Following strain construction, diploids were made and sporulation was induced to produce tetrads for dissection. The homolog strain was created by mating Y128 $\alpha$ to KHY252, the homeolog strain was created by mating Y597 to KHY252 and the homo-meolog strain was created by mating KHY252 to KHY258 (Table 1).

\section{Yeast containing a homeologous chromosome III in an otherwise all S. cerevisiae background were viable.}

The Y55 MAT $\alpha$ hybrid strain (Y597) has its native chromosome III replaced with chromosome III from S. paradoxus. The haploid strains (Y597, KHY246 and KHY258) 
containing this homeologous chromosome did not show any mutant phenotypes, suggesting that the substitution of chromosome III from S. paradoxus was functional.

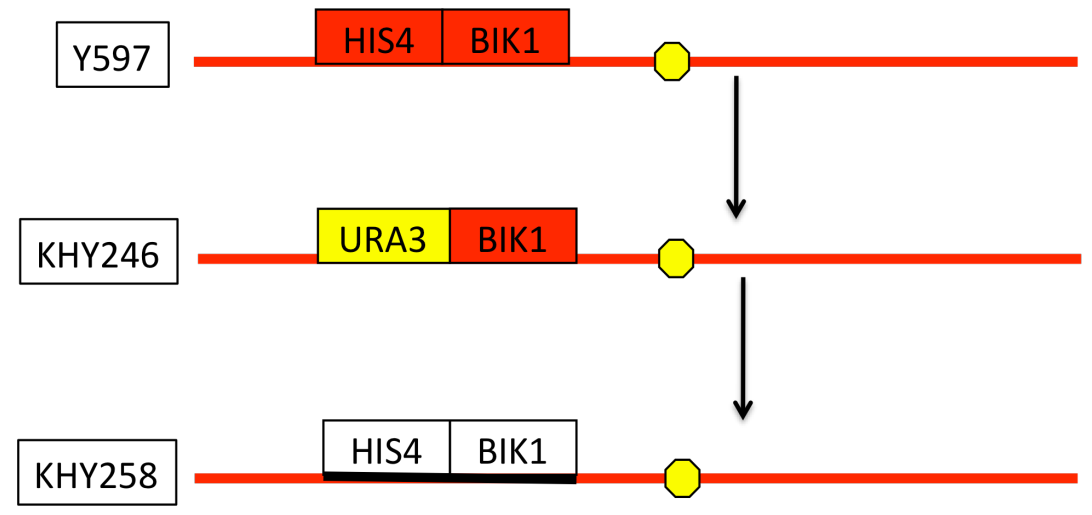

Figure 7: Construction of Homo-meolog strain. The construction of the Homo-meolog stain took place in two parts. First the S. paradoxus copy of HIS4 was removed and replaced with URA3, which was amplified from the plasmid pAG60. Then the URA3 insertion and native copy of BIK1 was removed and replaced with the S. cerevisiae copy of HIS4/BIK1, which was amplified off of isolated S. cerevisiae gDNA.

\section{The spore viability of the homeolog yeast strain is significantly reduced.}

Compared to the homologs, the homeologs show a decrease in overall spore viability. $73.5 \%$ of the tetrads from the homolog strain were 4 spore viables, while only $55.7 \%$ of the tetrads from the homeolog strain were 4 spore viables (Table 5, Fig. 9). This decrease in viability is not attributed to the substituted S. paradoxus chromosome III, as the haploid strain showed no visible growth defects. Furthermore, the homeolog strain shows the effects of failed meiotic events through an increase in 2 spore viable tetrads. Of the total tetrads analyzed, $5.7 \%$ were 2 spore viables in the homologs and $18.9 \%$ were 2 spore viables in the homeolog strain (Table 5, Fig. 9). The results of the chi-square analysis show that the observed differences in spore viability patterns between the homologs and the homeologs is statistically significantly different at $\mathrm{p}<0.01$. 
Table 5: Tetrad Analysis of Diploid Strains

\begin{tabular}{ccccccc|ccccc}
\hline \multirow{2}{*}{ Strain } & \multirow{3}{*}{$\begin{array}{c}\# \\
\text { Analyzed }\end{array}$} & \multicolumn{4}{c}{ Spore Viability } & \multicolumn{5}{c}{ Overall Score (\%) } \\
\cline { 3 - 13 } & $4: 0$ & $3: 1$ & $2: 2$ & $1: 3$ & $0: 4$ & $4: 0$ & $3: 1$ & $2: 2$ & $1: 3$ & $0: 4$ \\
\hline Homolog & 106 & 78 & 16 & 6 & 4 & 2 & 73.5 & 15.1 & 5.7 & 3.8 & 1.9 \\
Homeolog & 122 & 68 & 23 & 23 & 6 & 2 & 55.7 & 18.9 & 18.9 & 4.9 & 1.6 \\
Homo-meolog & 135 & 91 & 27 & 13 & 2 & 2 & 67.4 & 20.0 & 9.6 & 1.5 & 1.5 \\
\hline
\end{tabular}

Spore Viability

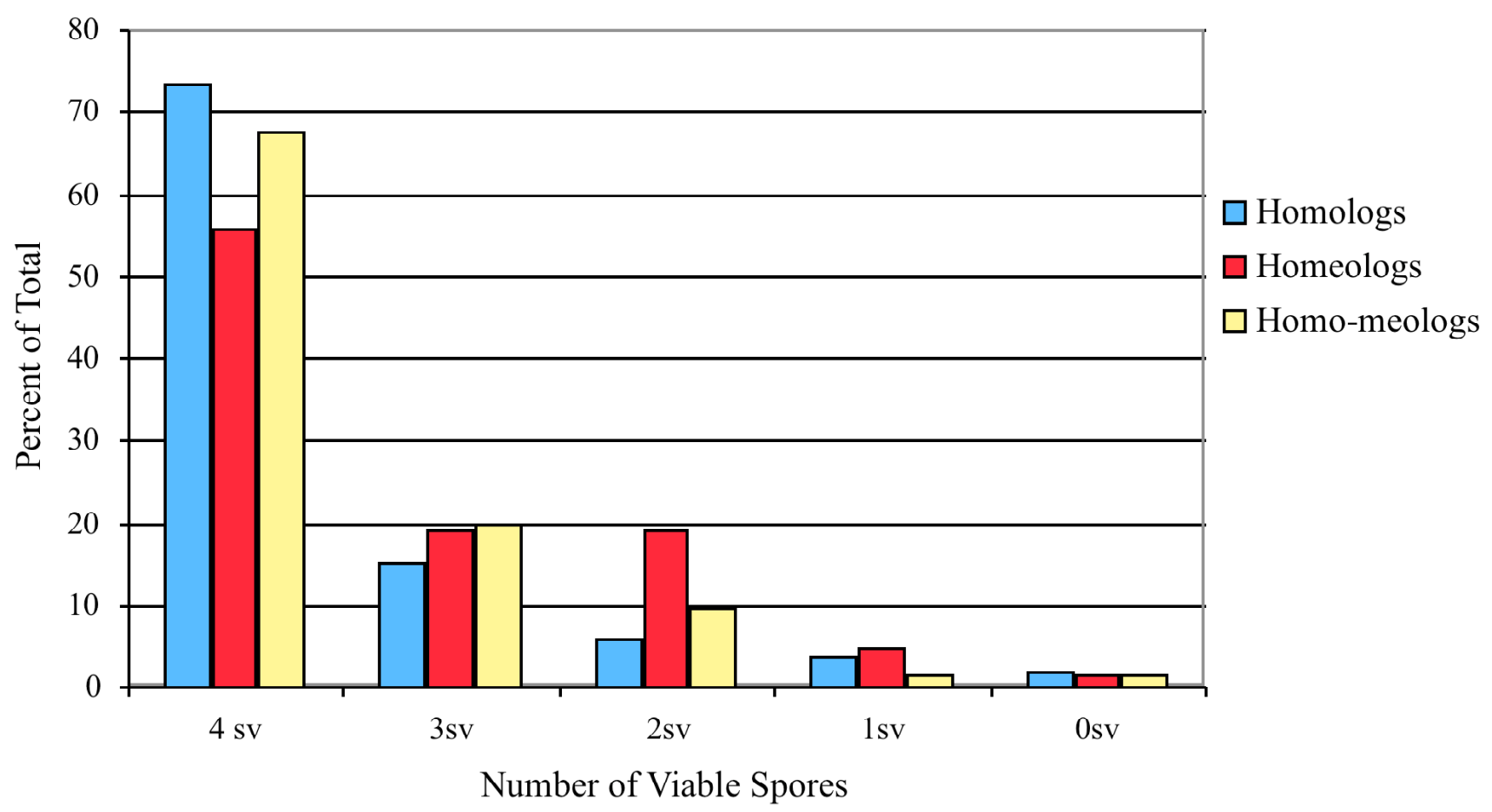

Figure 8: Spore Viability. Distribution of spore viability patterns for all constructs.

The homeolog strain 2 spore viable tetrads show a high degree of non-maters

Analysis of the 2 spore viable tetrads from the homeolog strain shows that 19 of the 232 spore viable tetrads appear to be non-maters, meaning they do not positively mate with either mating type during testing (Table 5). This is further evidence that these 2 spore viable tetrads are the result of non-disjunction event of chromosome III and not simply random spore death. 


\begin{tabular}{ccc}
\hline Strain & Maters & Non-maters \\
\hline Homolog 2 spore viables & 0 & 6 \\
Homeolog 2 spore viables & 4 & 19 \\
Homo-meolog 2 spore viables & 2 & 10 \\
\hline
\end{tabular}

The spore viability of the homo-meologs is slightly recovered, compared to the homeologs.

Compared to the homologs, the homo-meologs show an increase in overall spore viability. The homolog and homeolog strains showed $73.5 \%$ and $55.7 \% 4$ spore viable tetrads respectively. The homo-meolog strain however, showed $67.4 \% 4$ spore viable tetrads (Table 5, Fig. 9). The homo-meolog strain appears to shows the effects of crossover assurance through an increase in 4 spore viable tetrads. These tetrads may be the result of proper disjunction events due to recombination events occurring at the HIS4 hot spot inserted homology. Also, the proportion of 2 spore viable tetrads was rescued in the homo-meolog strain. While $18.9 \%$ of the homeolog tetrads were 2 spore viables, only 9.6\% of the homo-meolog tetrads were 2 spore viables (Table 5, Fig. 9). The results of the chi-square analysis show that the observed differences in spore viability patterns between the homologs and the homo-meologs is not statistically significantly different $(\mathrm{p}<0.01)$.

\section{Gene conversion events were doubled in the homo-meologs at HIS4.}

The extremely high incidence of NPD tetrads in the KAN-LEU interval spanning HIS4 observed in the homo-meologs in our data set suggests potentially extreme effects of crossover assurance (Table 10). Of the total tetrads analyzed, 6.4\% and $1.4 \%$ showed evidence of gene conversion in the homologs and homeologs respectively (Table 7). The quantity of observed gene conversion events was increased, relative to both the homologs and homeologs, to $12.6 \%$ in the homo-meolog strain (Table 7). We would have predicted 
however, that the appearance of these NPD segregation patterns would be accompanied with high levels of gene conversion at the HIS4 hot spot. However, we did not observe as much gene conversion as we would have initially predicted. Regardless, we did see a 2 fold increase in the number of gene conversion events from the homologs to the homomeologs that is statistically significant $(\mathrm{p}<0.05)$.

Table 7: Gene Conversion at HIS4

\begin{tabular}{ccccc}
\hline \multirow{2}{*}{ Strain } & \multicolumn{4}{c}{ Gene Conversion Pattern } \\
\cline { 2 - 5 } & $3: 1$ & $1: 3$ & $\%$ Total & Total n \\
\cline { 2 - 5 } Homologs & 5 & 0 & 6.4 & 78 \\
Homeologs & 0 & 1 & 1.4 & 68 \\
Homo-meologs & 8 & 6 & 12.6 & 91 \\
\hline Table 8: Gene Conversion at KAN & \multicolumn{5}{c}{} \\
\hline Strain & $3: 1$ & $1: 3$ & $\%$ Total & Total n \\
& 2 & 5 & 8.9 & 78 \\
Homologs & 1 & 1 & 2.9 & 68 \\
Homeologs & 2 & 3 & 5.5 & 91 \\
Homo-meologs & \multicolumn{5}{c}{}
\end{tabular}

Table 9: Gene Conversion at LEU2

\begin{tabular}{ccccc}
\hline Strain & \multicolumn{4}{c}{ Gene Conversion Pattern } \\
\cline { 2 - 5 } & $3: 1$ & $1: 3$ & $\%$ Total & Total $\mathrm{n}$ \\
Homologs & 0 & 0 & 0 & 78 \\
Homeologs & 0 & 0 & 0 & 68 \\
Homo-meologs & 1 & 1 & 2.2 & 91 \\
\hline
\end{tabular}

Table 10: Gene Conversion at MAT

\begin{tabular}{ccccc}
\hline Strain & \multicolumn{4}{c}{ Gene Conversion Pattern } \\
\cline { 2 - 5 } & $3: 1$ & $1: 3$ & $\%$ Total & Total $\mathrm{n}$ \\
Homologs & 0 & 0 & 0 & 78 \\
Homeologs & 0 & 0 & 0 & 68 \\
Homo-meologs & 1 & 2 & 3.2 & 91 \\
\hline
\end{tabular}




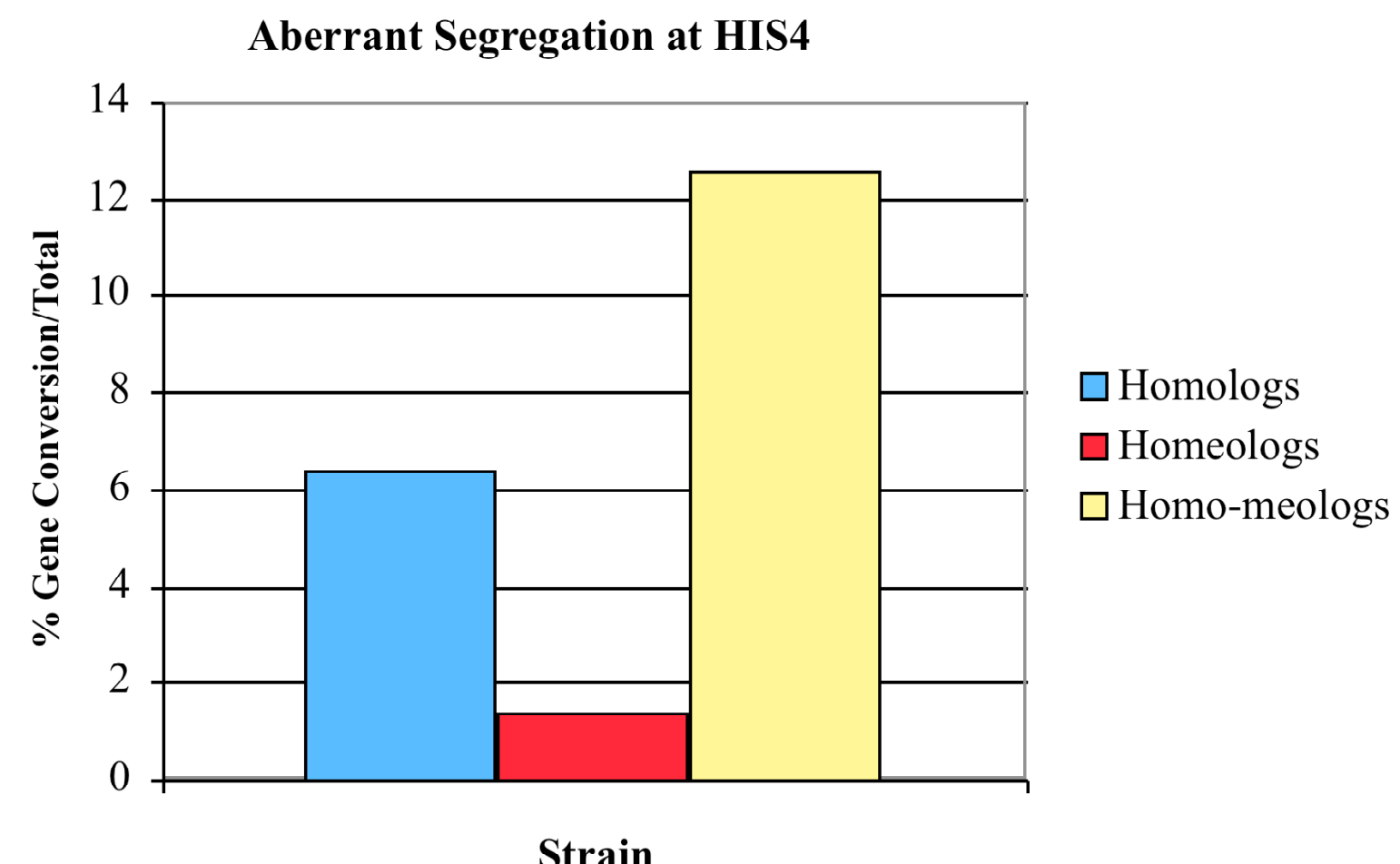

Figure 9: Gene Conversion at HIS4. Gene conversion events counted as any non 2:2 segregation pattern.

\section{Recombination rates are altered in the presence of homeologs and homo-meologs.}

The frequency of crossover events was measured in all three constructs, across the same intervals, in order to visualize the effects of crossover assurance. The recombination frequency within each of the three intervals was determined through examination of marker segregation patterns in tetrads. To analyze crossover patterns, only those tetrads with 4 viable spores were used. For comparisons, the homologs were used as a control for wildtype levels of crossing over along the entire length of the chromosome since this was the only strain that had two completely homologous copies of chromosome III. All the markers used to detect cross over events were located along chromosome III (Fig. 6), 
which spanned from YCL047C (position of KAN) through MAT. This accounts for approximately $49.4 \%$ of the entire physical length of the chromosome.

The frequency of crossing over was significantly increased in the KAN-LEU interval of the homo-meolog strain relative to both the homologs and homeologs (Table 10, Fig. 11). The map distance was $32.0 \mathrm{cM}$ in the homologs, evident of a high degree of crossing over. The map distance was significantly reduced in the homeologs $(3.57 \mathrm{cM})$, representing a substantial reduction in crossover events. The map distance for that same interval was $62.0 \mathrm{cM}$ for the homo-meologs, which is not only a restoration of wildtype levels but also almost a 2 fold increase in recombination. This increase is statistically significant $(\mathrm{p}<0.05)$.

The same increase in crossover frequency was also observed in the neighboring interval, between LEU-MAT in the homo-meolog construct. The map distance observed in the homologs for the LEU-MAT interval was $18.3 \mathrm{cM}$, and again this value was significantly decreased in the homeologs to $1.47 \mathrm{cM}$. Surprisingly however, the map distance for the LEU-MAT interval in the homo-meolog strain was $73.0 \mathrm{cM}$, indicative of completely un-linked genes (Table 10, Fig. 11). This implies a much higher frequency of crossing over in the homo-meolog strain compared to both the homologs and homeologs. 


\begin{tabular}{|c|c|c|c|c|c|c|}
\hline \multirow{2}{*}{ Strain } & \multirow{2}{*}{ Interval } & \multicolumn{4}{|c|}{ Count } & \multirow{2}{*}{$\begin{array}{c}\text { Map } \\
\text { Distance } \\
(\mathrm{cM})\end{array}$} \\
\hline & & $\mathrm{PD}$ & NPD & TT & Total & \\
\hline \multirow{2}{*}{ Homolog } & Kan-Leu & 24 & 0 & 43 & 67 & 32.0 \\
\hline & Leu-Mat & 45 & 0 & 26 & 71 & 18.3 \\
\hline \multirow{2}{*}{ Homeolog } & Kan-Leu & 54 & 0 & 4 & 58 & 3.57 \\
\hline & Leu-Mat & 66 & 0 & 2 & 68 & 1.47 \\
\hline \multirow{2}{*}{ Homo-meolog } & Kan-Leu & 19 & 8 & 59 & 86 & 62.0 \\
\hline & Leu-Mat & 12 & 10 & 56 & 78 & 73.0 \\
\hline
\end{tabular}

\section{Comparative Crossover Frequency}

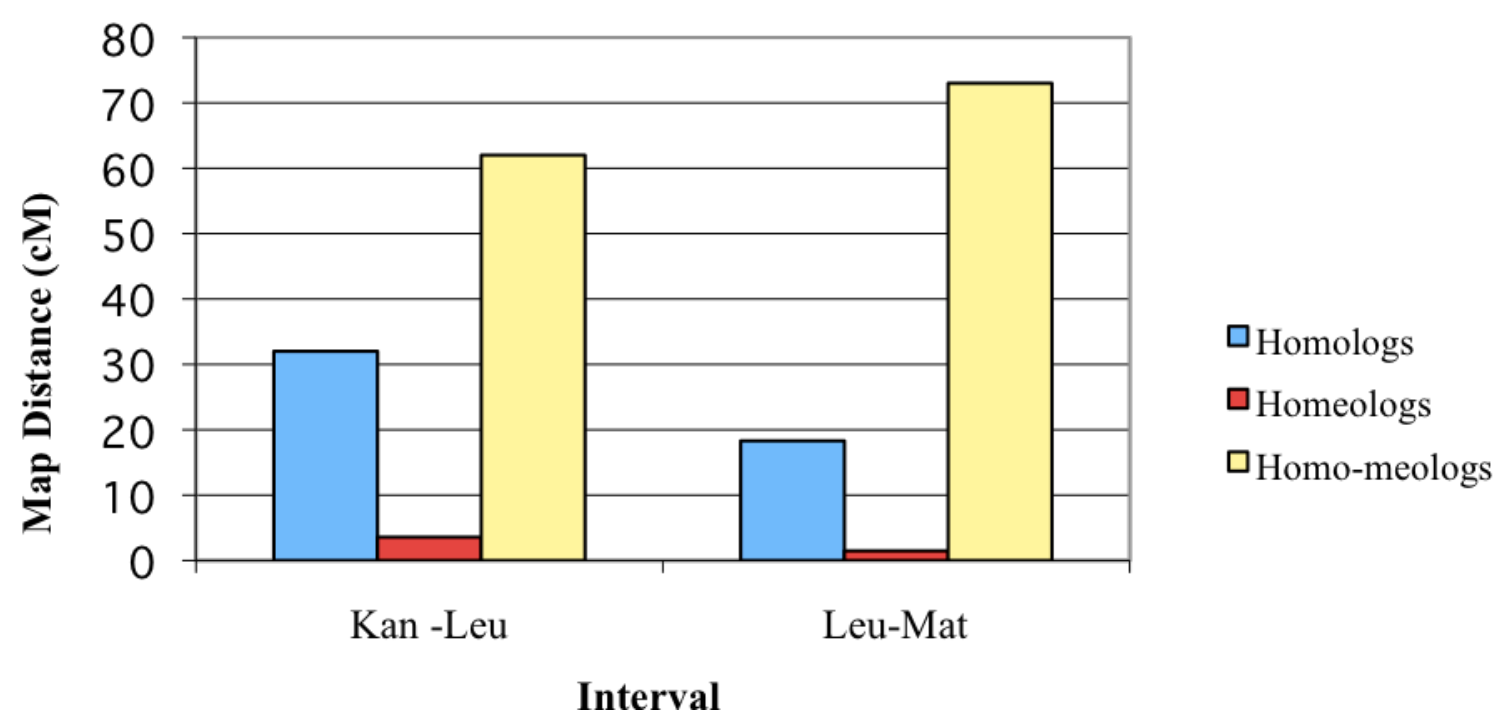

Figure 10: Comparative Crossover Frequency. Crossover data by interval was used to determine the map distances in each construct. Map distance was calculated using the Perkins equation (1947).

Crossing over in KAN-LEU did not influence crossing over in LEU-MAT in the homologs and homo-meologs but did in the homeologs.

Given the unexpected increase in crossing over that was observed in the LEU-MAT interval, questions arose about a potential positive influence of crossing over from the 
KAN-LEU interval on crossing over in the LEU-MAT interval. Several Fishers exact tests were run (Table 12) and the results indicate that the only strain in which crossing over in KAN-LEU positively affects crossing over in LEU-MAT is the homeologs.

Table 12: Crossover Occurrence Correlation

\begin{tabular}{c|ccccc}
\hline \multirow{2}{*}{ Strain } & \multicolumn{4}{c}{ Crossover Occurrence } \\
\cline { 2 - 5 } & Kan-Leu & Leu-Mat & Both & Neither \\
\hline Homologs & 19 & 9 & 11 & 6 \\
Homeologs & 1 & 5 & 3 & 67 \\
Homo-meologs & 11 & 12 & 47 & 5 \\
\hline
\end{tabular}

\begin{tabular}{ccc}
\hline Homologs & Crossover in Kan-Leu & No Crossover in Kan-Leu \\
\hline Crossover in Leu-Mat & 11 & 9 \\
No Crossover in Leu-Mat & 19 & 6 \\
\hline
\end{tabular}

*2-Tail: $\mathrm{p}$-value $=0.204$

\begin{tabular}{ccc}
\hline Homeologs & Crossover in Kan-Leu & No Crossover in Kan-Leu \\
\hline Crossover in Leu-Mat & 3 & 5 \\
No Crossover in Leu-Mat & 1 & 67 \\
\hline
\end{tabular}

*2-Tail: $\mathrm{p}$-value $=0.003$

\begin{tabular}{ccc}
\hline Homo-meologs & Crossover in Kan-Leu & No Crossover in Kan-Leu \\
\hline Crossover in Leu-Mat & 47 & 12 \\
No Crossover in Leu-Mat & 11 & 5 \\
\hline
\end{tabular}

*2-Tail: $\mathrm{p}$-value $=0.500$ 


\section{Discussion}

Despite being vital to the reproductive and genetic success of an organism, the regulation of recombination remains poorly understood (Lien et al., 2000). The results of this study have, however, contributed to the pool of information, which will ultimately help in determining the mechanism of crossover assurance.

We first took a broad look at the effects of our defined region of homology on crossover assurance by analyzing the spore viability patterns of all three constructs. In yeast, detailed analysis of meiotic products is simplified by the fact that all four spores of meiosis are packaged together in a tetrad. Also, in yeast, a cell that does not receive at least one copy of each chromosome is not viable and therefore because of this requirement we are able to assay chromosome segregation patterns. Due to the intimate correlation between chromosome segregation and crossing over, these patterns are also indicative of crossing over behaviors.

In the homologs, we observed a high incidence of four spore viable tetrads $(73.5 \%)$ with a decrease in 3, 2, 1, and 0 spore viable tetrads (Table 5). This pattern represents a typical distribution, such that the majority shows evidence of proper disjunction (4 spore viable) with some small amount of background random spore death accounting for the non-4 spore viable tetrads.

Homeologous chromosomes however, appear to present a challenge to crossover formation and subsequently inhibit proper chromosomal segregation. In yeast, mutations that eliminate crossovers result in decreased spore viability due to and increase in nondisjunction events during meiosis I (Maxfield Boumil et al., 2003). In the present study, the difficulty homeologous chromosomes experience when forming crossovers is evidenced by the increase in 2 spore viable tetrads, typical of non-disjunction events 
(Table 5, Fig. 8). These non-disjunction events are presumably the direct result of the nonhomologous chromosomes (III) failure to undergo crossing over. Hybrid organisms show increased vigor due to their high heterozygosity, however they trade this with decreased fertility (Lorenz et al., 2002). This reduction in fertility is likely a reflection of improper meiotic chromosome pairing. Chromosomes of hybrid yeast strains do not enable homologous pairing during meiosis (Lorenz et al., 2002). Lorenz et al. further observed significant non-homologous pairing between $S$. cerevisiae and S.paradoxus hybrids, which was attributed to insufficient homologous sites for recombination initiation (2002). It has also been proposed that when recombination events, such as crossovers, are initiated in regions of inadequate homology, the mismatch repair system is triggered and the recombination event is eliminated (Hunter et al., 1996).

As seen in the results, the homeologs show a statistically significant increase in 2 spore viable tetrads (from 6 to 23) compared to the homologs (Table 5, Fig. 8). Furthermore, approximately $80 \%$ of these 2 spore viable tetrads are also non-maters (Table 6). This is additional evidence that these spores are the result of non-disjunction at chromosome III because these spores are disomic for chromosome III. The non-maters contain two copies of chromosome III, which is the location of the mating type gene (MAT). Since they have two copies of the MAT locus they act as if they were diploids and therefore do not mate.

We hypothesized that a segment of homology present in otherwise nonhomologous chromosomes would positively impact spore viability. Indeed, our results showed that the homo-meologs exhibited a recovered spore viability pattern compared to the homeologs (Table 5, Fig. 8). Moreover, viability patterns in the homo-meologs were 
not statistically different $(\mathrm{p}<0.01)$ from the wild type homolog pattern. This recuperated viability pattern is potentially caused by the ability of the homo-meologs to form a crossover due to the region of shared homology at HIS4. The formation of a crossover in that region appears to be sufficient enough to allow for proper tension between the chromosomes, necessary for correct segregation. We therefore conclude that a small region of homology between chromosomes is enough to at least partially rescue spore viability patterns.

Next we wanted to look at the effects of our defined region of homology on crossover assurance more directly. To do this we initially looked at the incidence of gene conversion events in all constructs. We were particularly interested in gene conversion events happening in our region of interest, HIS4. The allele of HIS4 used contains an ATC instead of an ATG, which results in a poorly repaired C-C mismatch. This mismatch occurs during the strand invasion step of recombination. Analysis of PMS events at the HIS4 locus revealed that $\mathrm{C} / \mathrm{C}$ mismatches were 3 times less effectively repaired compared to other point mismatches (Detloff et al., 1991).

The his4-ATC allele, in fact, has showed higher PMS events than any other his4 initiation site mutant alleles (Detloff et al., 1991). It has been proposed that the meiotic mismatch repair system evolved to remove errors caused by DNA polymerase, which infrequently create $\mathrm{C} / \mathrm{C}$ mismatches. Therefore $\mathrm{C} / \mathrm{C}$ mismatch may be able to evade repair mechanisms so effectively because there has been less selective pressure on the meiotic mismatch repair system to correct them because they are so infrequently caused by DNA polymerase (Detloff et al., 1991). This particular allele therefore, will allow us to actually 
visualize gene conversion events because this mismatch should produce a high frequency of PMS sectored colonies.

As expected, the quantity of gene conversion events varied among the three different constructs. The homolog strain showed a baseline level of gene conversion, approximately $6.5 \%$ of the total tetrads analyzed (Table 7, Fig. 9). These events were accounted for by reviewing the tetrad scoring sheets for non-mendelian (non 2:2) marker segregation rather than by observing PMS events. A study by Fan et al. (1995) reported approximately $16.7 \%$ PMS events of total for the same allele. These results however, were obtained in a different strain background and under different temperature conditions. The discrepancy between our results and those reported by Fan et al. (1995) is likely caused by an inability to observe PMS events as sectored colonies. The PMS colony phenotypes were difficult to distinguish due to poor replica printing and cell transfer and so they were not recorded. For future studies, careful observation of colony formation will be conducted, as well as comparison of known PMS sectored colonies to ensure proper gene conversion counts can be made.

Despite the observation issues, all strains were analyzed the same way therefore comparisons can still be made. The significant decrease in observed gene conversion events at HIS4 in the homeolog strain ( 1.5\%) (Table 7, Fig. 9) correlates well with the observed decrease in spore viability patterns. Given that the homeolog strain experiences problems segregating chromosome III, likely due to the lack of recombination events, it is expected that the frequency of gene conversion would also be decreased. Although DSBs may occur in the homeologs, those breaks are probably repaired off the sister and therefore will not result in a gene conversion event. However, as predicted, the homo-meolog strain 
shows a significant increase in gene conversion events $(\sim 12.5 \%)$ relative to both the homologs and homeologs (Table 7, Fig. 9). Interestingly, we not only observed a recovery of gene conversion events but in fact a 2-fold increase, which is statistically significant ( $p$ $<0.05)$.

It is reasonable to infer that the increase in gene conversion events in the homomeologs is due to an increase in the number of DSBs in the homologous region. Because the majority of DSBs will occur in the regions of homeology and are therefore not likely resolved to form a crossover, those DSBs cannot contribute to the requirement for the obligate crossover. Therefore, the region of homology that can produce a viable crossover appears to receive more DSBs in order to meet the requirement. This suggests that DSB formation is regulated as part of the crossover assurance mechanism.

Finally we analyzed the actual crossover frequency changes in the three constructs. Analysis of crossover frequencies yielded some expected results as well as unanticipated outcomes. The homology present in the homo-meolog strain impacted the action of crossover assurance. In the Kan-Leu interval surrounding our homologous insert, a significant increase in crossing over was observed (Table 11, Fig. 10). The genetic map of the homologs (Fig. 11) shows a map distance of $32.0 \mathrm{cM}$ between the Kan and Leu markers, while the homeolog map distance for the same interval is only $3.57 \mathrm{cM}$. The homo-meolog map distance for the Kan-Leu interval was $62.0 \mathrm{cM}$, a significant increase from both the homologs and homeologs $(\mathrm{p}<0.05)$. These results support the idea that homology plays some role in the crossover assurance mechanism. 

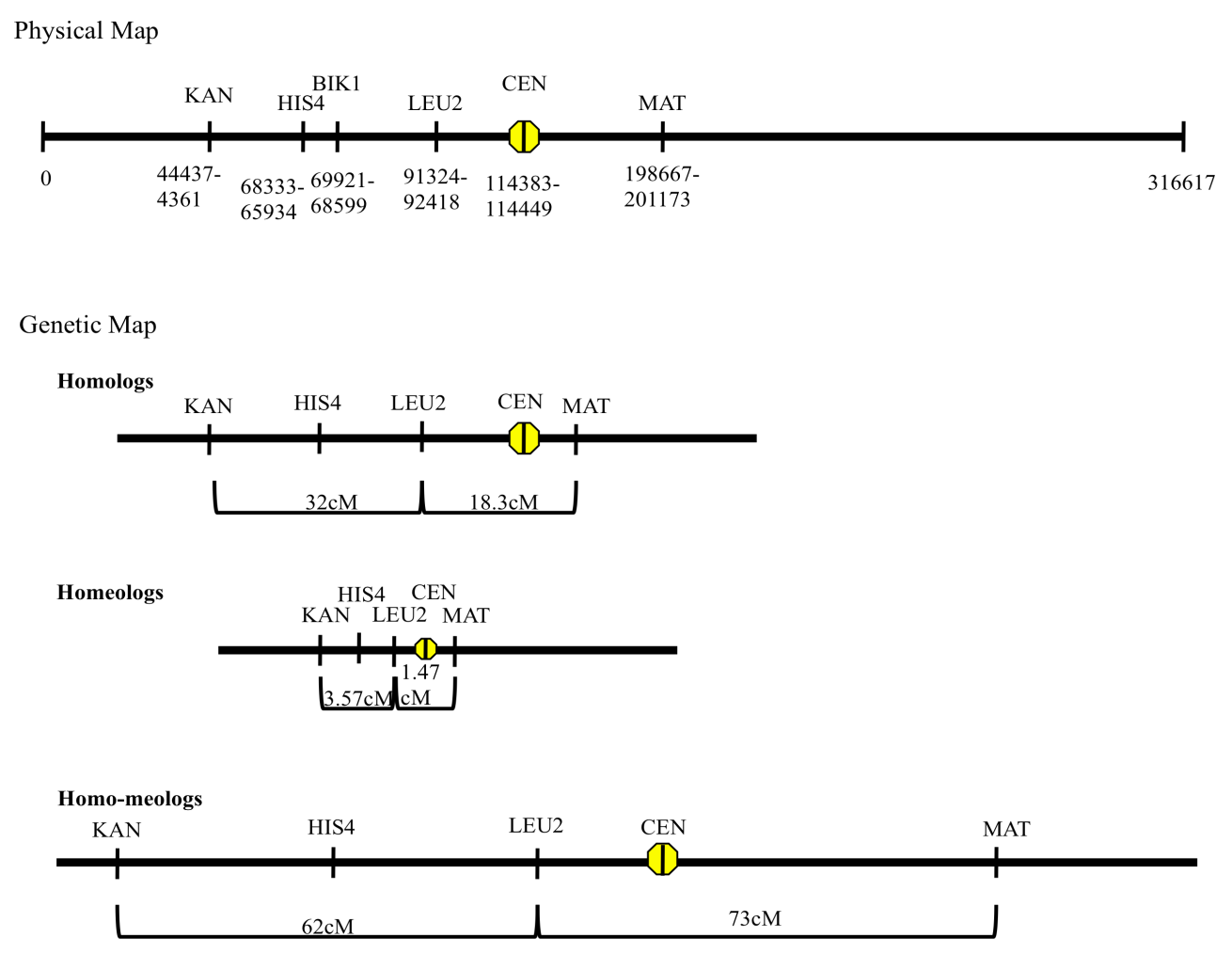

Figure 11: Physical and Genetic Map of Chromosome III. Physical map of S.cerevisiae chromosome III as determined by the base pair position of each marker. The genetic map was determined by crossover frequency data for each of the three constructs.

High levels of crossing over were also observed in the region outside of our homologous insertion in the Leu-Mat interval. This interval is genetically identical to the same interval in the homeolog strain (Fig. 6) and therefore should exhibit the same levels of crossing over. This, however, was not observed. Specifically, the genetic map distance for that interval was $1.47 \mathrm{cM}$ in the homeologs and $73.0 \mathrm{cM}$ in the homo-meologs (Table 11, Fig. 10). This high value map distance suggests completely unlinked genes and an indication of a substantial amount of crossing over. Not only was the recombination rate high, but many (10) NPD tetrads were observed, indicating the occurrence of double crossover events, which are normally very rare (Table 11). 
In conjunction with our observed results, this study leads us to the possibility that perhaps the barrier to recombination is not the inability of homeologous chromosomes to produce a crossover from a DSB, but in fact an inability to properly undergo pairing and synapsis. Pairing and synapsis is potentially required for efficient crossover formation. Perhaps the region of homology inserted at HIS4/BIK1 was sufficient enough to restore proper pairing and synapsis between the otherwise homeologous chromosome III and this effect spread slightly down the chromosome (Fig. 12). If this is true, we may expect to see a decrease in the crossing over frequencies farther away from the homologous insertion. To better evaluate if this is true, we need to conduct several tests. First we need to be able to assay the amount of crossing over that occurs at the far ends of chromosome III. The placement of makers in locations i, v and vi (Fig. 4) should allows us to illuminate the underlying origin of our unanticipated crossover frequency data.

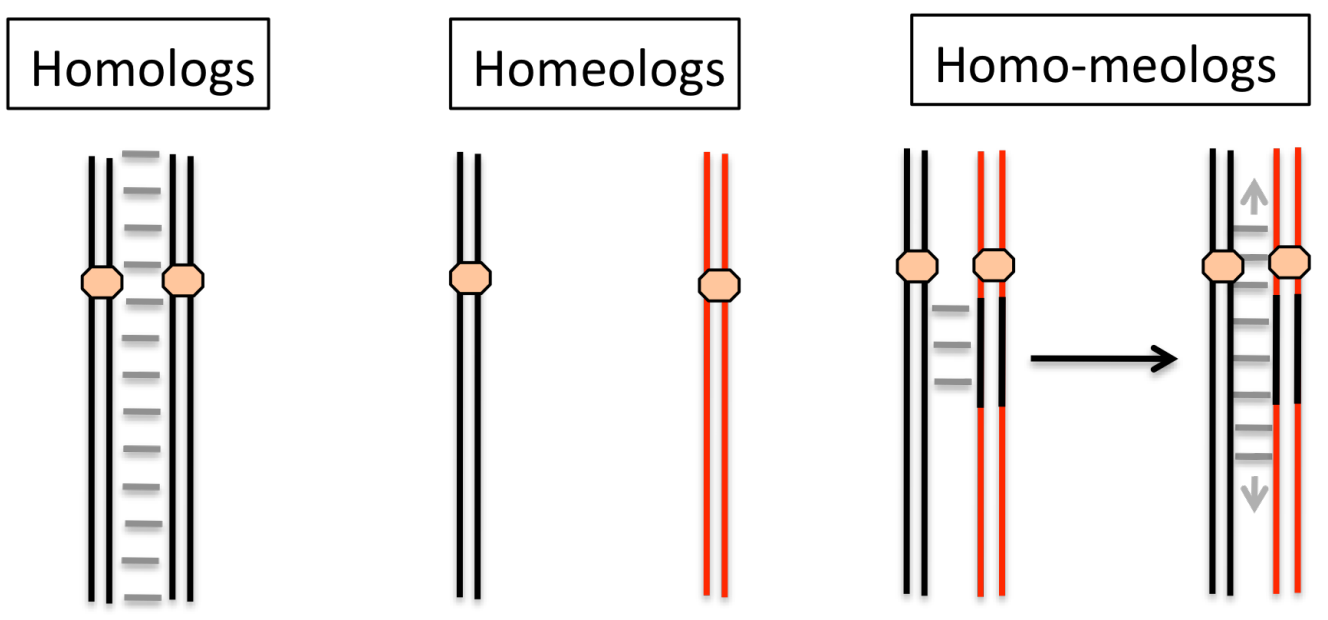

Figure 12: Pairing and Synapsis Variations between strains. Given the total homology present between the homologs, pairing and synapsis should not pose a problem for these chromosomes. However, because of the lack of homology present between the homeologs, they may not be able to find one another to pair and therefore are also unable to synapse.

The homo-meologs present an interesting hybrid of these two scenarios. The region of homology present may be sufficient enough to allow for pairing and synapsis in that region and potentially the subsequent spread of the synapsis slightly outside the region of homology. 
It has long been known that different regions of chromosomes vary in the amount and frequency of recombination events they experience. For example, telomeric regions have been shown to exhibit below average numbers of recombination events (Broman et al., 1998). Particularly high rates of recombination have been reported in both the mouse and human pseudoautosomal regions (PARs) (Rappold, 1993). The recombination rate between the $\mathrm{X}$ and Y PAR has been reported to be 10-20 times higher than the predicted rate (Schmitt et al., 1994). The obligate crossover has been further evidenced by an approximate $50 \%$ recombination rate during male meiosis in the PAR between the $\mathrm{X}$ and Y chromosomes (Burgoyne, 1982).

The existence of double crossovers within the region of limited homology in the PAR has been the subject of much debate. Recent studies, however, have reported some double recombinants, suggesting that PARs not only experience higher than average recombination rates but also receive double crossover events. In one such study conducted on the mouse PAR, an almost 7-fold increase in crossing over was observed in the PAR compared to the genome-wide average. Furthermore, in conjunction with the increase in crossover frequency, several incidences of three point crosses, which are indicative of double crossover events and decreased interference, were observed (Soriano et al., 1987). Taken together, these results can be viewed as possible effects of crossover assurance. The homo-meolog construct we created in order to study the effects of crossover assurance, mimics that of the PAR. Our homo-meolog strain mimics the $\mathrm{X}$ and $\mathrm{Y}$ chromosomes, with our inserted region of homology between the S. cerevisiae and S. paradoxus chromosome III acting like a PAR. 
If DSBs are independent of one another, the expected ratio of marker segregation in double crossover tetrads is 1 PD: 2 TT: 1 NPD. Therefore, the observed increase in NPD tetrads (Table 11) in the Leu-Mat interval of the homo-meologs was unexpected.

However, given the restraints on our homo-meolog system, it is plausible that the DSBs occurring are no longer independent of one another (Fig.13).

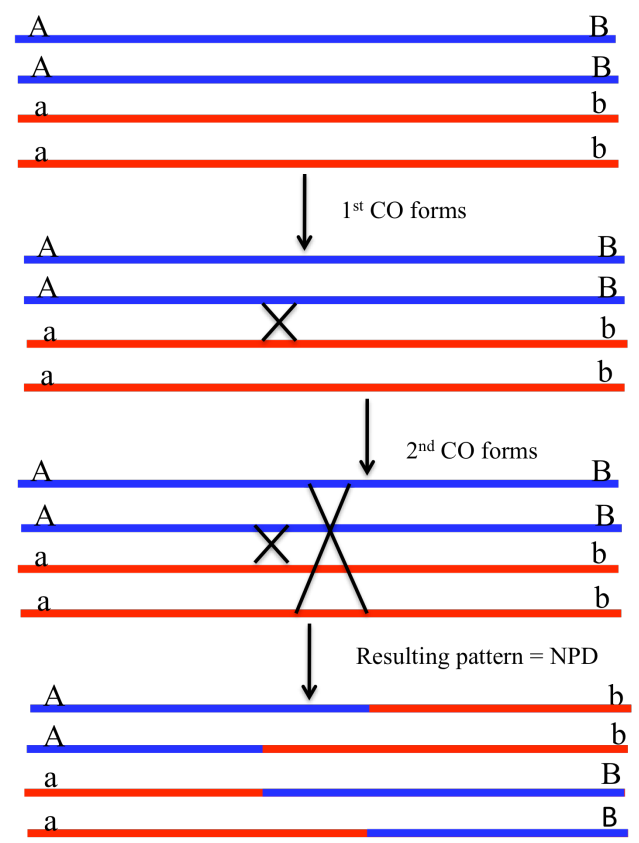

Figure 13: Double Crossover Formation and NPD Tetrads. In a system where there is limited homology, and given that at least one crossover must occur between each pair of chromosomes, DSBs which are the initiating events for CO may not be independent of one another. If one $\mathrm{CO}$ forms between two chromatids, the physical connection between them will prevent them from participating in another DSB and CO. This may then result in the formation of an NPD segregation pattern.

However, despite the appearance of double crossover events, it is arguable that typing errors cannot be completely ruled out as the cause for the observation of these rare recombination events (Schmitt et al., 1994). Our data set was checked multiple times over, to ensure these forms of error were not responsible for our results. Therefore, the only other conclusion that we can draw is that there are flaws in our strain construction that are skewing our analysis. More extensive genetic analysis by PCR and Southern blot needs to 
be performed on our homo-meolog strain to determine the exact placement of our region of homology and the markers surrounding it and to confirm integration at a single locus.

\section{Future Directions}

Once in place, however, our homo-meolog system will have numerous applications for studying the effects and mechanism of crossover control. The immediate next steps include the complete marking of the S. cerevisiae chromosome III and further PCR testing of the homo-meolog strain.

We will also be able to evaluate changes in meiotic machinery in the homo-meolog construct such as changes in DSB formation and Spo11 activity. Meiotic mutants can also be assayed. Fluorescence in situ hybridization (FISH) is a cytological technique used to identify chromosomes by use of their DNA sequences. This will be a critical tool to analyze the pairing and synapsis of the homeolog and homo-meolog strains. Also, Chromatin immunoprecipitation (ChIP) is yet another technique that can be used to analyze chromosome/protein interactions. This experimental tool can be used to specifically identify proteins that closely associate with genomic sequences. We would hope to use this technique to assay the changes in protein binding at the HIS4 locus between our homolog, homeolog and homo-meolog strains.

While this project remains ongoing and the story continues to unfold, we are optimistic about the usefulness of our system in advancing the search for the mechanics behind crossover control, specifically crossover assurance. 


\section{References}

Argueso, J., J. Wanat, Z. Gemici, E. Alani. 2004. Competing crossover pathways act during meiosis in Saccharomyces cerevisiae. Genetics. 168:1805-1816.

Baker, B., A. Carpenter, M. Esposito, R. Esposito, L. Sandler. 1976. The genetic control of meiosis. Annu. Rev. Genet. 10: 53-134.

Bascom-Slack, C., L. Ross, D. Dawson. 1997. Chiasmata, crossovers, and meiotic chromosome segregation. Advances in Genetics. 35: 253-84.

Borner, G., N. Kleckner, N. Hunter. 2004. Crossover/noncrossover differentiation, synaptonemal complex formation, and regulatory surveillance at the leptonene/zygotene transition of meiosis. Cell. 117: 29-45.

Broman, K., J. Murray, V. Sheffield, R. White, J. Weber. 1998. Comprehensive human genetic maps: individual and sex-specific variation in recombination. Am.J.Hum.Genet. 63: 861-869.

Burgoyne, P. 1982. Genetic homology and crossing over in the X and Y chromosomes of mammals. Hum Genet. 61: 85-90.

Chambers, S., N. Hunter, E. Louis, R. Borts. 1996. The mismatch repair system reduces meiotic homeologous recombination and stimulates recombination-dependent chromosome loss. Mol Cell Biol. 16:6110-6120.

Detloff, P., M. White, T. Petes. 1992. Analysis of a gene conversion gradient at the HIS4 locus in Saccharomyces cerevisiae. Genetics. 132:113-123.

Detloff, P., J. Sieber, T. Petes. 1991. Repair of specific mismatches formed during meiotic recombination in the yeast Saccharomyces cerevisiae. Mol Cel Biol. 11: 737-745.

Esposito, M. 1971. Postmeiotic segregation in Saccharomyces. Molec Gen Genetics. 111: 297-299.

Fan, Q., F. Xu, T. Petes. 1995. Meiosis-specific double-strand DNA breaks at the HIS4 recombination hot spot in the yeast Saccharomyces cerevisiae: control in cis and trans. Mol Cell Biol. 15: 1679-1688.

Gerton, J., J. DeRisi, R. Shroff, M. Lichten, P. Brown, T. Petes. 1999. Global mapping of meiotic recombination hotspots and coldspots in yeast Saccharomyces cerevisiae. PNAS. 97: 11383-11390

Hassold, T., P. Hunt. 2001. To err (meiotically) is human: the genesis of human aneuploidy. Nature. 2: 280-291. 
Hunter, N., S. Chambers, E. Louis, R. Borts. 1996. The mismatch repair system contributes to meiotic sterility in an interspecific yeast hybrid. EMBO J. 15: 1726-1733.

Jones, G., C. Franklin. 2006. Meiotic crossing-over: obligation and interference. Cell. $126 ; 246-248$.

Kaback, D., D. Barber, J. Mahon, J. Lamb, J. You. 1999. Chromosome size-dependent control of meiotic reciprocal recombination in Saccharomyces cerevisiae: the role of crossover interference. Genetics. 152:1475-1486.

Keeney, S., C. Giroux, N. Kleckner. 1997. Meiosis-specific DNA double-strand breaks are catalyzed by Spo11, a member of a widely conserved protein family. Cell 88: 375-84.

Kleckner, N. 2006. Chiasma formation: chromain/axis interplay and the role(s) of the synaptonemal complex. Chromosoma. 115: 175-194.

Kleckner, N. 1996. Meiosis: How could it work? Proc. Natl. Acad. Sci. 93: 8167-74.

Lien, S., J. Szyda, B. Schechinger, G. Rappold, N. Arnheim. 2000. Evidence for heterogeneity in recombination in the human pseudoautosomal region: high resolution analysis by sperm typing and radiation-hybrid mapping. Am J Hum Genet. 66: 557-566.

Lorenz, A., J. Fuchs, E. Trelles-Sticken, H. Scherthan, J. Loidi. 2002. Spatial organization and behavior of the parental chromosome sets in the nuclei of Saccharomyces cerevisiae x S. paradoxus hybrids. J. Cell Sci. 115: 3829-2835.

Mancera, E., R. Bourgon, A.Brozzi, W. Huber, L. Steinmetz. 2008. High-resolution mapping of meiotic crossovers and noncrossovers in yeast. Nature. 454: 479-485.

Mangs, A., B. Morris. 2007. The human pseudoautosomal region (PAR): origin, function and future. Curr Genet. 8: 129-136.

Martini, E., R. Diaz, N. Hunter, S. Keeney. 2006. Crossover homeostasis in yeast meiosis. Cell. 126; 285-295.

Maxfield Boumil R, B. Kemp, M. Angelichio, T. Nilsson-Tillgren, D.S. Dawson. 2003. Meiotic segregation of a homeologous chromosome pair. Mol Gen Genom. 268: 750-60.

Naumov, G., E. Naumova, R. Lantto, E. Louis, M. Korhola. 1992. Genetic homology between Saccharomyces cerevisiae and its sibling species $S$. paradoxus and S. bayanus: electrophoretic karyotypes. Yeast. 8: 599-612.

Nicklas, B. 1974. Chromosome segregation mechanisms. Genetics. 78: 205-13.

Papazian, H. 1952. The analysis of tetrad data. Genetics. 37: 174-188. 
Perkins, D. 1947. Biochemical mutants in the smut fungus Ustilago maydis. Genetics 34: 607- 626.

Porter, S., M. White, T. Petes. 1993. Genetic evidence that the meiotic recombination hotspot at the HIS4 locus of Saccharomyces cerevisiae does not require a site for symmetrically processed double-strand break. Genetics. 134: 5-19.

Rappold, G. 1993. The pseudoautosomal regions of the human sex chromsomes. Hum Genet. 92: 315-324.

Roeder, G. 1997. Meiotic chromosomes: it takes two to tango. Gene Dev. 11: 2600-2621.

Roig, I., S. Keeney. 2008. Probing meiotic recombination decisions. Develop Cell. 15: 331-332.

Ruderfer, M. 2006. Population genomic analysis of outcrossing and recombination in yeast. Nature. 38: 1077-1081.

Schmitt, K. et al. 1994. Multipoint linkage map of the human pseudoautosomal region, based on single-sperm typing; do double crossovers occur during male meiosis. Am J Hum Genet. 55: 423-430.

Shubochkina, E., T. Nielsen, T. Nissoon-Tillgren. 2001. Meiotic crossing-over in the regions of homology between homeologous chromosomes V. Yeast, 18: 1173-1183.

Soriano, P., E. Keitges, D. Schorderet, K. Harbers, S. Gartler, R. Jaenisch. 1987. High rate of recombination and double crossovers in the mouse pseudoautosomal region during male meiosis. Proc Natl Acad Sci USA. 84: 7218-7220.

Szostak, J., T. Orr-Weaver, R. Rothstein, F. Stahl. 1983. The double-strand-break repair model for recombination. Cell. 33: 25-35.

White, J., K. Lusank, S. Fogel. 1985. Mismatch-specific post-meiotic segregation frequency in yeast suggests a heteroduplex recombination intermediate. Nature. 315: 350352.

Zeyl, C. 2000. Budding yeast as a model organism for population genetics. Yeast. 16: 773784. 